\title{
Wireless Sensing and Communication Capabilities from In-Core to a Monitoring Center
}

\section{August 2020}

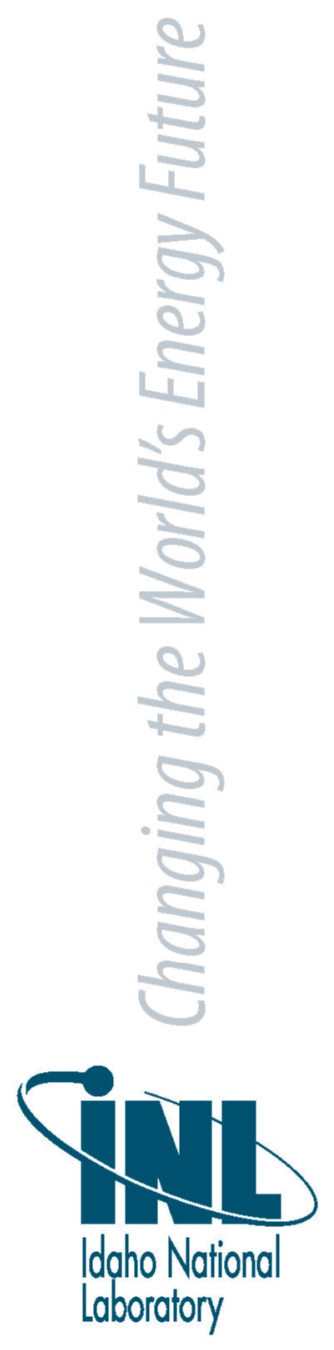

James A. Smith, Idaho National Laboratory

Chengying "Cheryl” Xu, North Carolina State University

Yiming Deng, Michigan State University

Koushik A. Manjunatha, Idaho National Laboratory

Vivek Agarwal, Idaho National Laboratory 


\section{DISCLAIMER}

This information was prepared as an account of work sponsored by an agency of the U.S. Government. Neither the U.S. Government nor any agency thereof, nor any of their employees, makes any warranty, expressed or implied, or assumes any legal liability or responsibility for the accuracy, completeness, or usefulness, of any information, apparatus, product, or process disclosed, or represents that its use would not infringe privately owned rights. References herein to any specific commercial product, process, or service by trade name, trade mark, manufacturer, or otherwise, does not necessarily constitute or imply its endorsement, recommendation, or favoring by the U.S. Government or any agency thereof. The views and opinions of authors expressed herein do not necessarily state or reflect those of the U.S. Government or any agency theof. 


\section{Wireless Sensing and Communication Capabilities from In-Core to a Monitoring Center}

James A. Smith, Idaho National Laboratory

Chengying "Cheryl" Xu, North Carolina State University Yiming Deng, Michigan State University

Koushik A. Manjunatha, Idaho National Laboratory

Vivek Agarwal, Idaho National Laboratory

June 2020

Idaho National Laboratory
Idaho Falls, Idaho 83415

http://www.inl.gov

Prepared for the

U.S. Department of Energy

Office of Nuclear Energy

Under DOE Idaho Operations Office

Contract DE-AC07-05ID14517 
Page intentionally left blank 


\section{SUMMARY}

There is an opportunity to achieve significant cost reduction if electrical cables are replaced by wireless technologies in current nuclear power plants (NPPs) and are part of advanced reactor designs. Wireless technology can also provide in-core opportunities by significantly reducing the number of penetrations in the pressure vessel, reducing the cost and complexity of sensor installation, and increasing the efficiency of current and advanced reactors. Unlike in other deployment scenarios for industrial environments, operators need centralized control over all the networks. Centralized control will reduce implementation costs, provide single-point control, enable the monitoring of network devices, improve security, and enhance connectivity.

Micro-sensors that simultaneously monitor temperature and pressure within NPP fuel rods will help foster preventative actions during abnormal operating conditions. This could avert accidents and enable expedient development of accident-tolerant fuels. A novel micro-sensor suite $(\sim \mathrm{mm})$ to simultaneously measure multiple parameters such as temperature, strain, pressure, and neutron/gamma flux inside fuel rods is being developed for use in reactors. The necessary communication architecture is also being developed to transmit measurement signals from the core to the plant's data cloud or control room.

A three-tier strategy has been developed to support wireless transmission of in-core measurements to the control room or secure cloud platform for control, analytics, and decision-making purposes.

1. In-core: data signal from in-core to outside the pressure vessel within the containment building

2. Containment building: data signal from inside the containment building to the outside, and on into the balance of the plant network

3. Balance of the plant network: information related to structures, systems, and components on the balance of plant side transmitted to the data cloud and control room.

This report presents a review of wireless sensing and communication systems for use both within a reactor core and elsewhere. The communication technology is advantageous in terms of compensating for network equipment failures and adverse data transmission conditions. Wireless technology will significantly increase the resiliency of plants' network systems. The wireless system naturally provides multi-path transmission capability and data redundancy.

Ceramic-based high-temperature sensors that are wireless and passively powered will be integrated into communication networks to enable delivery of in-core measurements to a cloud database and dataprocessing center. The system will provide real-time sensing for in-core nuclear applications subject to high-temperature, high-pressure, high-radiation fluxes and oxidation challenges. This requires sensors that are robust with respect to high-radiation and high-temperature environments. The goal is to collect real-time information from sensors located throughout the reactor, so the reactor core and fuel elements within can be monitored cost-effectively via precise, localized measurements. The high-temperature- and radiation-resistant sensors will be suitable for a variety of reactor types (e.g., light-water reactors [LWRs], gas reactors, etc.). This report, however, focuses on LWR applications, since the advanced reactors are in the initial design phase and subject to significant design changes. The sensing techniques in this report focus on electromagnetic (EM) techniques, and conductive coolants such as molten salt or liquid metal afford significant challenges for EM transmission.

Polymer-Derived Ceramic (PDC) sensor materials seem particularly promising. One advantage of PDC sensors is their multi-sensing capability for simultaneously measuring temperature, pressure, strain, and other parameters. The sensors will be frequency multiplexed thanks to micro-patch antennas designed to resonate within different frequency bands. An advanced communication system will be developed in which multiple sensors can be interrogated directly and in parallel. An appropriate amount of radio frequency (RF) power can be radiated using the transceiver antenna to actuate the passive radio frequency 
identification (RFID) sensor node at an estimated minimum required power level of $0.363 \mathrm{~mW}$ at $5 \mathrm{GHz}$. The range of the RFID sensor to the transceiver antenna is expected to be on the order of meters. Instrumentation to demodulate, process, and digitize the data will be located in a more hospitable environment within the containment building.

The complexity and layered construction of the containment wall will make it extremely difficult to transmit wireless signals out of the containment building. The steel, gastight lining acts as a Faraday cage, making standard RF transmission impractical. The reinforced concrete is highly attenuative to ultrasound. While communication techniques may be developed independently for each separate layer, combining the wall layers drastically increases the difficulty of transmission. The difficulty in transmitting signals out of the containment building provides ample research opportunities and is outside of the scope of this report.

Although it is appealing to keep the transmission system wireless, multi-layer containment walls significantly impede through-wall signal transmission. Even if through-wall technology is successful, this cutting-edge technology still needs to compete with wired/cable transmission on both a practical and an economic basis. The major impediments to using cabled transmission are the costs of installing/routing the cables and their vulnerability to mishaps caused during normal plant operations and maintenance. For applications in the containment building, the impediments to using cables are significantly reduced. The run for a single cable would be relatively short — on the order of $500 \mathrm{ft}$ - and the potential for mishap is reduced by being within a limited space indoors.

The reliability of wired cables is impressive. Electrical cables have performed safely for longer than the 40-year planned lifetime of the typical NPP, with few age-related cable failures recorded. It would be difficult for other forms of instrumentation to match the operating lifetimes and low maintenance afforded by electrical cables. A single cable's $30-\mathrm{GHz}$ communication bandwidth would be sufficient to transfer all of the required monitoring data. Adding a single cable to the existing electrical trays and feedthroughs should be feasible and would make for a reasonable compromise. The ultimate decision will be based on the engineering tradeoffs necessary in making the upgrades. For advanced reactors still being designed, the possibilities for wireless are unlimited, as the transmission systems can be creatively built into the reactor structures.

To meet the plant's goals, wireless sensing and communication system development will be phased according to the following stages. The individual technologies, RFID sensors and infrastructure, and the balance of the plant communication network are currently at Technology Readiness Level -4 (TRL-4). Each technology will require modification to integrate with the other technologies.

- RF sensors will need to be integrated into the RFID infrastructure.

- The RFID infrastructure will be integrated with the balance of the plant network.

- TRL-4 testing will be conducted for the fully integrated wireless sensing and communication system.

At every stage, the sensing system will be tested to ensure measurement quality and the efficiency of the communication systems. From the initial sensor stage consisting of lab RFID instrumentation to TRL4 testing of the fully integrated system, all subsystems and integrated systems will be quality-tested.

As part of the path forward, the RFID infrastructure will be designed to multiplex multiple sensors. The multiplexed system with at least six multiplexed sensors will be designed and benchmarked against industry-standard thermocouples. The passive RFID sensor will be located at a range of $0.1-1 \mathrm{~m}$ from the transceiver antenna submerged in water to test the RF interrogator's communication range and sensing capability to simulate in-core conditions. Temperature testing will occur in furnaces located at the various facilities where development and integration take place. The fully integrated system will be tested at TRL4 using an autoclave within INL's High Temperature Test Laboratory (HTTL). 
Although the great challenge is to take in-place and real time measurements inside fuel rods within an NPP, the goal of the implementation plan is to develop a fully integrated wireless sensing and communication system to TRL-4. This system will be installed at HTTL, and the signal relay will be connected to INL's wireless link. The measurement data will then be transmitted to a cloud database housed in another building several blocks away. As part of the system testing, end-to-end performance, network delay, and the quality of the received data will be analyzed using INL's distributed antenna system test bed located in the Human System Simulation Laboratory. The RFID sensors will also be benchmarked against traditional wired sensors. 
Page intentionally left blank 


\section{ACKNOWLEDGEMENTS}

This report was made possible through funding by the U.S. Department of Energy's Nuclear Energy Enabling Technologies Program. We are grateful to Suibel Schuppner at the U.S. Department of Energy as well as Craig Primer and Pattrick Calderoni at Idaho National Laboratory for championing this effort. We thank John M. Shaver and Lisa Wells at INL for technical editing and formatting of the document as well as Deepak Kumar, a Ph.D. student at Michigan State University. 


\section{CONTENTS}

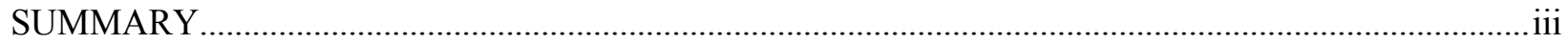

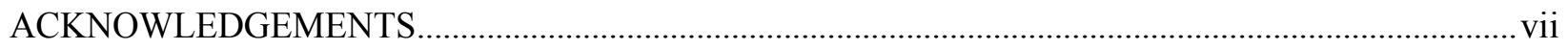

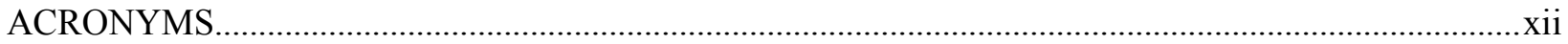

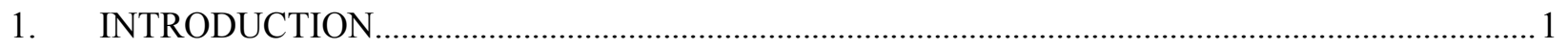

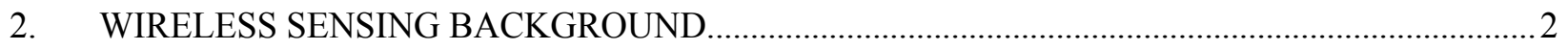

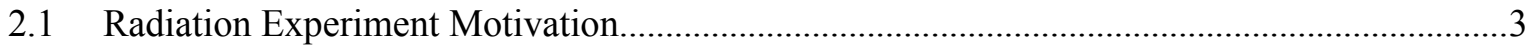

3. PASSIVE MONITORING AND WIRELESS COMMUNICATION ARCHITECTURE................... 3

4. RFID SENSOR RUGGEDIZATION FOR HARSH ENVIRONMENTS.......................................... 8

4.1 Plan for Field-Deployable Sensor and Signal Transmission.......................................................

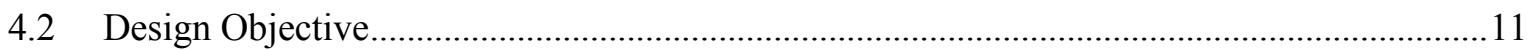

4.3 Properties of Polymer-Derived Ceramics...............................................................................12

4.3.1 Unique and tunable semiconducting behavior for temperature measurement............ 12

4.3.2 High piezoresistivity for pressure measurement....................................................... 13

4.3.3 Excellent thermo-mechanical properties at up to $1500^{\circ} \mathrm{C}$ for high-temperature

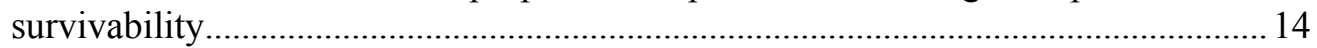

4.3.4 Probable high radiation resistance for irradiation survivability............................... 15

4.3.5 Micro-fabrication capability to ensure low-cost mass production of the proposed

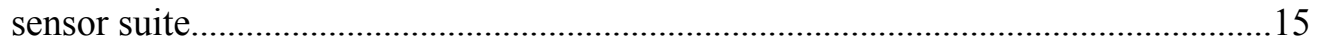

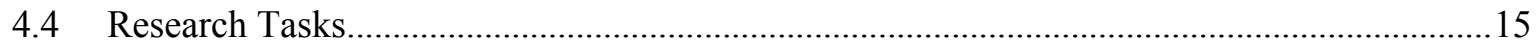

4.4.1 Task 1: Developing PDC material with desired electrical properties for accurate sensor measurement............................................................................................ 15

4.4.2 Task 2: Designing and fabricating the sensor suite for simultaneous temperature and

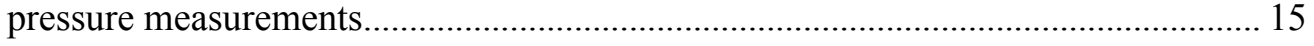

4.4.3 Simultaneous temperature and pressure measurements..............................................16

4.4.4 Signal conditioning system and wireless transmission............................................ 17

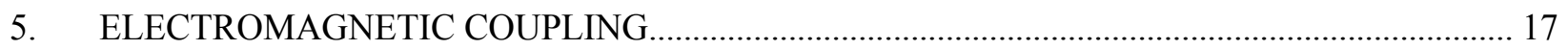

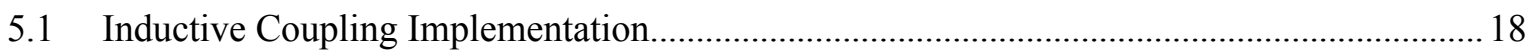

5.2 Radio Frequency Modulator Implementation............................................................................ 20

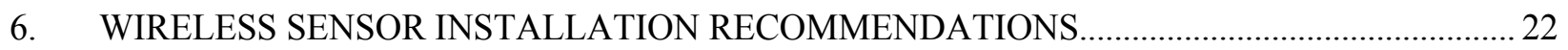

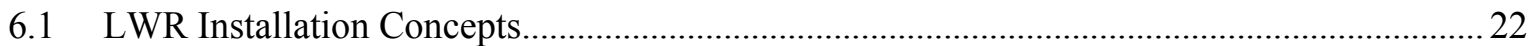

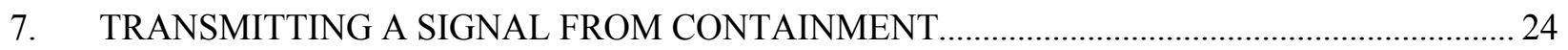

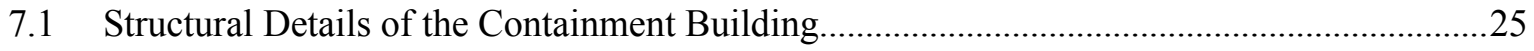

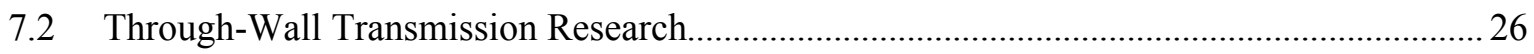

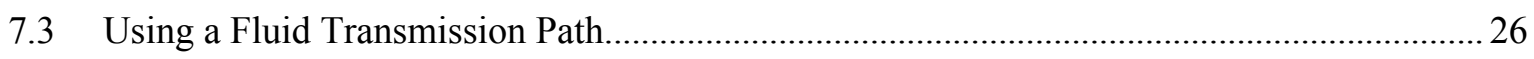

7.4 Electrical Cable for Containment Transmission........................................................................26 
8. TRANSMITTING A SIGNAL FROM THE BALANCE OF THE PLANT ....................................27

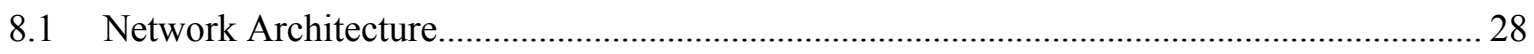

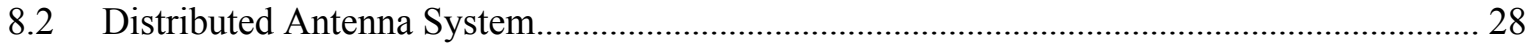

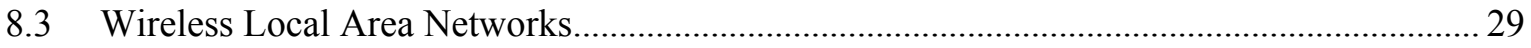

8.4 Industrial, Scientific, and Medical Band Technologies.............................................................30

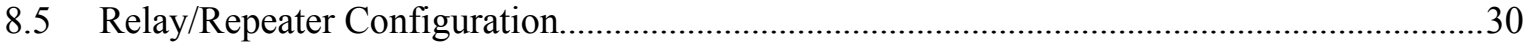

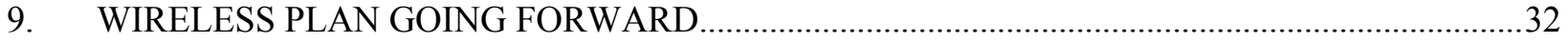

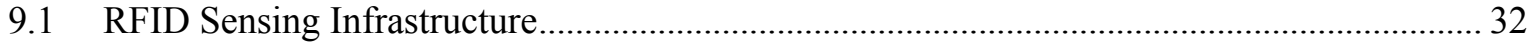

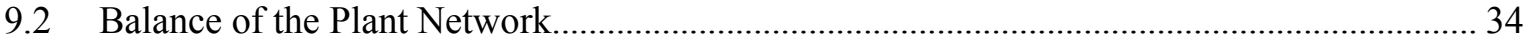

10. WIRELESS RESEARCH AND DEVELOPMENT ROADMAP .................................................. 35

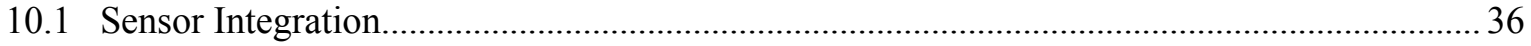

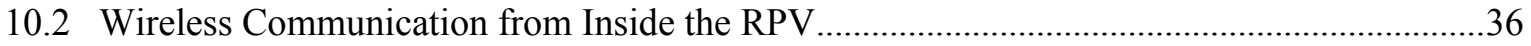

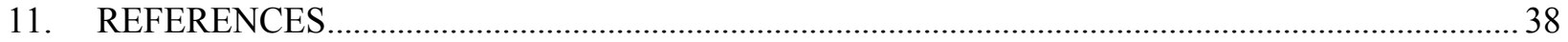

\section{FIGURES}

Figure 1. Three-tier strategy to support wireless transmission of in-core measurements to the control room or secure cloud platform for control, analytics, and decision-making purposes. The wireless system naturally provides multiple transmission path capability and data redundancy...

Figure 2. Battery-less wireless sensing and communication network for NPPs. Numerous sensing nodes installed around the facility over the next several decades can be interrogated wirelessly

Figure 3. Fully assembled 3D-printed pressure sensor with component annotation and dimensions.

Figure 4. (Left) Pressurized pipeline setup with a harmonic RF sensor tag for real-time wireless pressure monitoring. (Right) Wirelessly measured phase change due to applied pressure.

Figure 5. Frequency change of temperature and luminescence sensors with (A) linear fit and (B) second-order polynomial fit.

Figure 6. (Left) Frequency spectrum of the demodulated sensor data. (Right) Wireless demonstration with 9-dBm transmitted power at $908 \mathrm{MHz}$, showing received sensor information in a time domain with a switching frequency of $6 \mathrm{kHz}(\sim 160 \mu \mathrm{s})$.

Figure 7. Schematic diagram of the LWR-2 fuel rodlet and capsule assembly for ATR irradiation. Dimensions are in inches.

Figure 8. Typical oxide fuel drop-in capsule configuration (zoom-in: the sensor suite is "hung" inside the rodlet by an electrical feedthrough in the cap)...

Figure 9. Schematic diagram showing the proposed sensor-transmitter system.

Figure 10. Processing steps for synthesizing polymer-derived ceramics $[21,22]$. 
Figure 11. Electrical conductivity of a PDC as a function of temperature.

Figure 12. Electrical conductivity of PDCs as a function of composition...................................................13

Figure 13. Electrical resistance as a function of applied stress [28] ......................................................... 14

Figure 14. Scanning electron microscope images of the surfaces of (a) typical silicon-based ceramics after heat-treatment in water vapor and/or NaCl-containing environments, (b) PDCs after annealing at $1400^{\circ} \mathrm{C}$ for $300 \mathrm{hrs}$ in $50 \% \mathrm{H}_{2} \mathrm{O}-50 \% \mathrm{O}_{2}$, and (c) PDCs after annealing at $1200^{\circ} \mathrm{C}$ for $50 \mathrm{hrs}$ in a $\mathrm{NaCl}$-containing environment [29]...

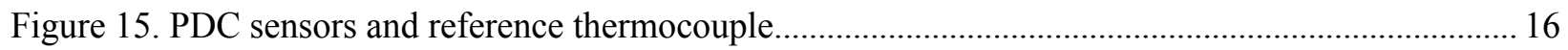

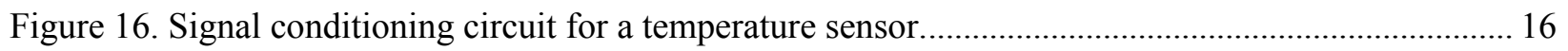

Figure 17. The inductive-sensor system design used in the MITR (redrawn from Carvajal et al.) [41]

Figure 18. The self-powered detector signal is used to modulate an AM transmitter built from VME components and transmitted to a data acquisition system outside the reactor (redrawn from [44]).

Figure 19. Experimental implementation of the wireless transmission system in the Breazeale Reactor, showing the external powering of the transmitters and the wired delivery of the SPD signal. The antennas were eventually replaced by cable connections due to intermittent signal transmission (redrawn from [41]).

Figure 20. Representative fuel-element schematic showing how the ends of the fuel rods are accessed. (Graphic redrawn courtesy of the U.S. Department of Energy.).

Figure 21. Schematic of the installation of the rod cluster assembly onto the fuel element, showing the EM shadowing of the fuel rod caps. The rod cluster control assembly shields flow orifices in the top nozzle from the transceiver located at the top of the reactor. However, the rod cluster does provide an opportunity to bring a transceiver closer to the fuel rods by routing it through the shaft of the control assembly. (Photo redrawn from Areva: https:/www.sfen.org/sites/default/files/public/atoms/files/2012markus_birkhofer_areva_the_importance_of_the_fuel_design.pdf.)...

Figure 22. Schematic of the installation of the top nozzle onto the fuel element, showing EM shadowing of the fuel rod caps. Flow orifices in the top nozzle provide possible EM transmission paths. (Photo redrawn from Areva: https://www.sfen.org/sites/default/files/public/atoms/files/2012markus_birkhofer_areva_the_importance_of_the_fuel_design.pdf.)...

Figure 23. Schematic of a boiling-water reactor, showing the containment building. Figure courtesy of NRC [51].

Figure 24. Envisioned DAS wireless network architecture for NPP automation.

Figure 25. DAS testbed installation at INL's Human System Simulation Lab. Left: MPROU, Right: BU

Figure 26. Schematic of an RF interrogator for wireless communication with the passive RF sensor node and the relaying of information to the balance of the plant network. 33

Figure 27. Data flow from the reactor core to the cloud using the balance of the plant network. 34

Figure. 28. Simplified model of a nuclear reactor pressure vessel and the eight battery-less sensing nodes integrated inside featuring radiation-hardened electronics and high- 


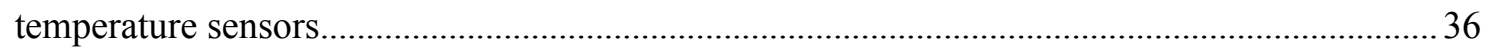

Figure 29. Acoustic interrogator for wirelessly communicating inside the RPV .......................................37

Figure 30. Architecture of the battery-less sensing node with a wireless acoustic communication system.

\section{TABLES}

Table 1. Comparison of different wireless technologies. 


\section{ACRONYMS}

$\mathrm{AE}$

AP

ATR

BSI

CCPT

DAS

EM

EU

HetNet

HTTL

IoT

ISM

LoRaWan

LTE

LWR

LWRS

MAC

MODEM

MPROU

MSU

MU

MU-MIMO

NPP

ODU
Antenna elements

Access points

Advanced Test Reactor

Base station interface

Capacitively coupled power transfer

Distributed antenna system

Electromagnetic

Expansion unit

Heterogeneous networks

High Temperature Test Laboratory

Internet of Things

Industrial, scientific, and medical

Long-range wide-area networks

Long-Term Evolution

Light-water reactor

Light-water reactor safety

Media Access Control

Modulator-demodulator

Mid-Power Remote Optic Unit

Michigan State University

Main unit

Multi-user multi-input multi-output

Nuclear power plant

Optical Distribution Unit 


$\begin{array}{ll}\text { OFDMA } & \text { Orthogonal frequency division multiple access } \\ \text { PDC } & \text { Poly-derived ceramic } \\ \text { QoS } & \text { Quality of service } \\ \text { RF } & \text { Radio frequency } \\ \text { RFID } & \text { Radio frequency identification } \\ \text { RPV } & \text { Reactor pressure vessel } \\ \text { RU } & \text { Remote unit } \\ \text { SDR } & \text { Software-defined ratio } \\ \text { SNR } & \text { Signal-to-noise ratio } \\ \text { SPD } & \text { Self-powered detector } \\ \text { TRL } & \text { Technology readiness level } \\ \text { VME } & \text { Vacuum micro-electronic } \\ \text { WLAN } & \text { Wireless local area networks }\end{array}$


Page intentionally left blank 


\section{Wireless Sensing and Communication Capability from In-Core to a Monitoring Center}

\section{INTRODUCTION}

Electrical cables are part of the structures, systems, and components that play a vital role in the safe and reliable operation of the current fleet of nuclear power plants (NPPs). Cable failures, though rare, are serious events needing to be prevented. Cables are designed to last many decades under normal operating conditions, and they generally do so. Cables are included in regularly scheduled tests for NPP systems and active devices. Degradation of electrical insulation and other cable components over the last 60 years is of crucial concern. Such degradation may cause failure in the installed cables and prevent NPPs from operating safely and reliably for another 40 years beyond their initial qualified operating life. The cost of laying and maintaining cables in NPPs is significant.

A typical NPP has more than 1,000 km of cables relating to power, control, instrumentation, etc., within the plant. It is not feasible to replace or inspect all these cables. Effective guidelines that rely on sampling and screening of cables have been put in place as part of ageing management plant and are evolving. Cables are tested based on accessibility, risk, history, and other factors. Because of the diverse array of cables and operating conditions, multiple tests must be performed to ensure a cable's reliability. It is imperative that degraded cables are accurately identified and repaired or replaced prior to the end of their safe operating lives. Ageing cable management programs include a database of cables selected - based on accessibility, risk, and environmental considerations - for testing and trending via the appropriate cable characterization technique.

Significant cost savings can be made if cables are replaced by wireless technologies in current NPPs and included as part of advanced-reactor designs. Wireless technology can also provide in-core opportunities by significantly minimizing the number of penetrations into the pressure vessel, reducing the cost and complexity of sensor installation, and increasing the efficiency of current and advanced reactors. A three-tier strategy is developed to support wireless transmission of in-core measurements to the control room or a secure cloud platform for control, analytics, and decision-making purposes:

- In-core: data signal from in-core to outside the pressure vessel within the containment building

- Containment building: data signal from inside the containment building to the outside, and on into the balance of the plant network

- Balance of the plant network: information transmitted to the data cloud and monitoring center

The core of this report is organized according to the three-tier wireless strategy outlined in Figure 1. A review of the motivation and background for wireless sensing is given, leading to a dialog on radio frequency identification (RFID) wireless measurement architecture. Polymer-derived ceramic (PDC) sensors designed for in-core sensing and used in the wireless measurement architecture are then described next. Alternate electromagnetic (EM) measurement techniques for communicating through metal containers are then discussed. Since the basics of RFID and EM sensing techniques have already been explored, an exemplary in-core light-water reactor (LWR) application will be covered as a way of transmitting in-core measurements out of the reactor pressure vessel. Once the measurement signal is outside the reactor vessel, the ensuing discussion encompasses research on how data can be transmitted out of the containment building. Once the signal is out of the containment building, the dialog focuses on the communication infrastructure for transmitting the measurement signals to the balance of the plant network and over to the NPP data cloud or control room. Since all the proposed technologies presented here are at Technology Readiness Level 4 (TRL-4), an integration plan will be discussed for fully integrating a wireless sensing and communication system. A novel self-powered ultrasonic communication system is also discussed for transmitting data through the pressure vessel wall. 


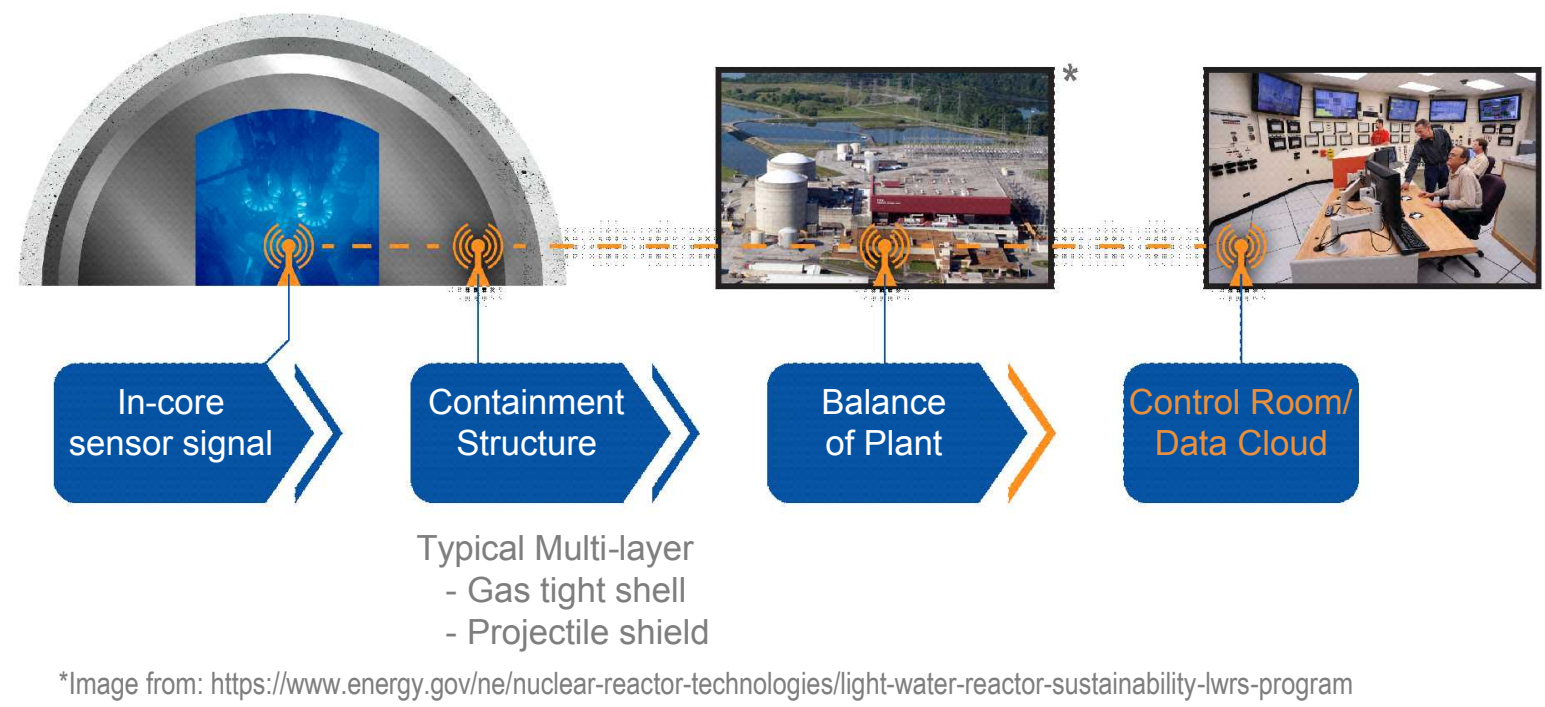

Figure 1. Three-tier strategy to support wireless transmission of in-core measurements to the control room or secure cloud platform for control, analytics, and decision-making purposes. The wireless system naturally provides multiple transmission path capability and data redundancy.

\section{WIRELESS SENSING BACKGROUND}

Process- and structural-monitoring systems are commonly used to assess the status and states of processes and structures during operations. Monitoring systems can be found in commercial processes, as well as in a number of common structures such as aircrafts, ships, and civil structures - not to mention nuclear reactors. Monitoring systems are tasked with gathering measurements from sensors mounted within the process or structure of interest. The gathered data are then mostly stored in a centralized database. To ensure that the data are reliably transmitted, monitoring systems generally use cabled wires to receive the signal transmissions from the sensors. Though cabled wires can be dependable transmission links, cable installation in processes and structures is costly and labor-intensive. When wired monitoring systems expand (increase in sensor number), their expenses can increase at a higher, non-linear rate.

To remove the inherent limitations in using wired sensing technologies, researchers are developing new technologies to transform process- and structural-monitoring methods. Wireless sensing [1-5] can advance the implementation of "smart" processes and structures by cost-effectively enabling the creation of large sensor networks. Wireless sensor development was originally driven by anticipated cost-related benefits. The abolition of long lengths of cabled wires in a process or structure keeps installation costs manageable. To effectively use sensors contained within a closed metal component under harsh conditions such as high temperatures, high pressure, high radiation, or limited volume, wireless transmission of data and power can be utilized. Concurrent transfer of data and power throughout the container volume will enable cost-effective sensor installation. The cost-effectiveness of wireless monitoring systems will enable large number of sensor node densities compared to tethered monitoring systems. The ability to have a larger number of sensors at more salient locations fosters enhanced condition-monitoring and state-of-health assessments for processes and structures. Wireless technology will significantly increase the resiliency of plants' networked communication systems and naturally provide multiple transmission path capability and data redundancy.

Active wireless sensors that rely on internal energy sources for power restrict the application of wireless monitoring. Power consumption is a significant constraint for wireless sensors powered by batteries. Battery-based wireless sensor nodes are expensive, require timely maintenance, and are complex for in situ monitoring. For nuclear applications, areas exist in which batteries are a hazard. 
Therefore, a battery-less (passive) wireless sensing technology is needed. Currently, RFID sensing is the only wirelessly powered communication technology. RFID sensors receive radio energy transmitted in the form of a continuous wave from a remote interrogator, and they reflect the identification and sensing information back to the receiver. The battery-less nature of RFID sensors makes them economical for large-scale integration, maintenance-free, and less complex, with compact, flexible packaging.

\subsection{Radiation Experiment Motivation}

Micro-sensors that simultaneously monitor the temperature and pressure of the fuel rod cladding in nuclear systems will enable preventative actions during abnormal operating conditions, thus averting accidents and fostering development of accident-tolerant fuels. The main challenge in developing such sensors is that they must be able to operate for long periods under harsh reactor conditions, including high temperatures (up to $1500 \mathrm{~K}$ ), high pressure, and high levels of radiation damage. Current sensing materials suffer severe structural and functional degradations when exposed to high-temperature and radiation in a reactor environment, thus losing their sensing capability. A novel micro-sensor suite ( $\mathrm{mm}$ ) that utilizes PDC materials for simultaneously measure multiple parameters such as temperature, strain, pressure, and neutron/gamma flux inside the fuel rod cladding in nuclear reactors is being considered.

Design times for nuclear fuels are legendary amongst the scientific community. The main culprit for the long design times is the "cooling off period," measured in years, that irradiated nuclear fuels must go through before being handled. The fuel sits in a "cooling" pool until the fuel rods can be transferred to a nuclear facility for post-irradiation examination of the fuel. The transfer process and hot cell coordination also add months just to be able to open the fuel cladding to characterize the fuel performance. To speed up the design process, a series of experiments are planned far in advance so the fuel can be "cooked" under various conditions and "looked" at years later. Thus far, this is the only approach available to fuel designers. However, unexpected events and transients do occur in the reactor and within the experiment. These unplanned conditions can have unknown consequences on the fuel. It will take fuel designers an average of 2.5 years to cut open the fuel cladding and determine what happened, then another year to plan and redo the experiment. All in all, it will take six years to complete the study or perhaps reach the decision to do without the data entirely.

Even a simple temperature measurement would be helpful for understanding fuel performance, as temperature plays a large role in the lifetime of a fuel assembly. However, current in-pile temperaturesensing technologies such as melt wires and silicon carbide bars reside within the fuel cladding and are not accessed until the cladding is cut open 2.5 years later. The proposed micro-sensor suite for simultaneous temperature and pressure measurements addresses the limitations of current state-of-the-art "cook \& look" measurement technology by utilizing wireless sensors to provide real-time in-pile data collected at a sampling rate of at least $10 \mathrm{~Hz}$. Real-time monitoring of the fuel will dramatically reduce the development time and costs for new fuels. If researchers know the fuel did not reach the desired operating temperature, experimental/reactor parameters may be adjusted; or the researchers can start planning a new experiment before the fuel is removed. These steps would save at least 2.5 years on the development timeline. Wireless signal transfer can also be used to increase operating efficiencies, reduce run time, and ascertain state awareness of fuel health under harsh operating conditions in commercial power reactors.

\section{PASSIVE MONITORING AND WIRELESS COMMUNICATION ARCHITECTURE}

Next-generation advanced monitoring systems for NPPs should be developed based on a battery-less RFID wireless communication architecture [1-6]. The passive RFID system does not require an onboard powering mechanism, yet flawlessly communicates ID information at a wireless range of up to tens of meters. The same fundamental principle can be applied to wireless communication of real-time sensing 
information from position "A" to position "B."

A number of parameters (e.g., pressure, temperature, current, flow, strain, vibration, radiation, etc.) must be continuously monitored for the safe, reliable operation of an NPP [7]. However, current monitoring systems are either wired or active (battery-operated) wireless, as well as unsustainable and in need of routine maintenance. Battery-less wireless sensing and communication is the holy grail in terms of being an optimized monitoring solution that also reduces deployment time and operational costs. Moreover, it allows for dense sensor integration, leading to high-resolution monitoring. Figure 2 shows the passive wireless sensing and communication architecture.

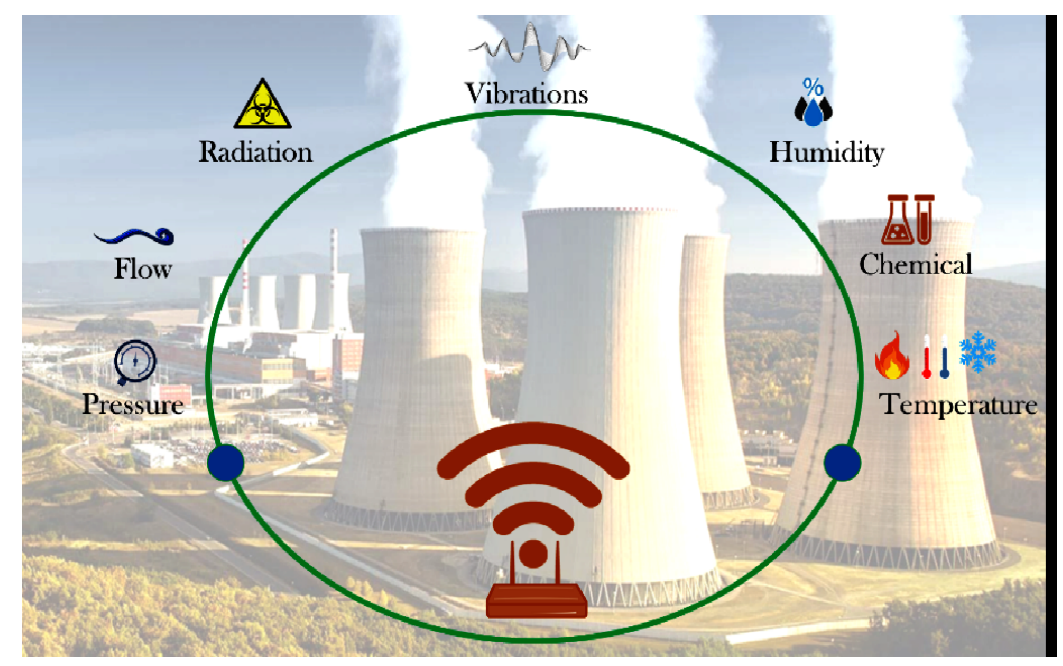

Figure 2. Battery-less wireless sensing and communication network for NPPs. Numerous sensing nodes installed around the facility over the next several decades can be interrogated wirelessly.

Integration of any sensor with an RFID tag proved very challenging, requiring decades of research to create a practical sensing system for potential use in an industrial facility [8-9]. The primary challenge was to reduce the sensor's power requirements and develop a coupling (sensing plus communication) mechanism that staged the requirement of power efficiency. The low power requirement is directly proportional to the longer communication range of battery-less systems. A power-efficient, single- or multi-sensor integrable platform is designed to communicate the ID and sensing information when applied for monitoring purposes. The information is fundamentally transferred by manipulating the amplitude, phase, or frequency of the radio signal via a process called "modulation." In this report, two topologies are discussed for battery-less wireless communication, demonstrating power-efficient phaseand frequency-modulation techniques for passive radio-frequency (RF) tags and illustrating the capabilities of single- or multi-sensor integration.

First topology [10]: An efficient, passive, wireless harmonic communication system is shown for realtime monitoring of pressurized pipelines. A pressure sensor is fabricated using additive manufacturing techniques, and a harmonic RFID tag operates at the fundamental frequency $\left(\mathrm{f}_{\mathrm{o}}\right)$ of $2 \mathrm{GHz}$, shifting the phase of the back-reflected RF signal according to the applied pressure. Power-efficient phase modulation with virtually no losses is achieved using a hybrid coupler-based phase shifter to efficiently reflect back the incoming signal using an end-coupled reactive impedance element/sensor. The phase delay introduced by the reactive element is doubled with the second harmonic communication, increasing the sensitivity by a factor of two. The concept of harmonic backscattering is exploited to reduce the effects of multi-path interference and self-jamming, as well as to improve the signal-to-noise ratio (SNR). 

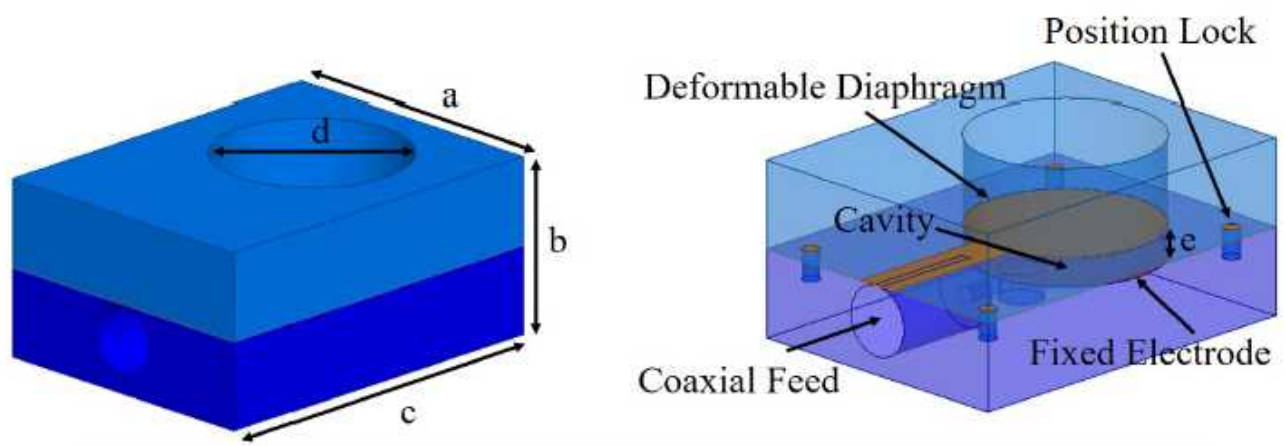

\begin{tabular}{|c|c|c|c|c|c|}
\hline Parameter & $\mathrm{a}$ & $\mathrm{b}$ & $\mathrm{c}$ & $\mathrm{d}$ & $\mathrm{e}$ \\
\hline Dimensions $(\mathrm{mm})$ & 10 & 6 & 13 & 6.6 & 1 \\
\hline
\end{tabular}

Figure 3. Fully assembled 3D-printed pressure sensor with component annotation and dimensions.
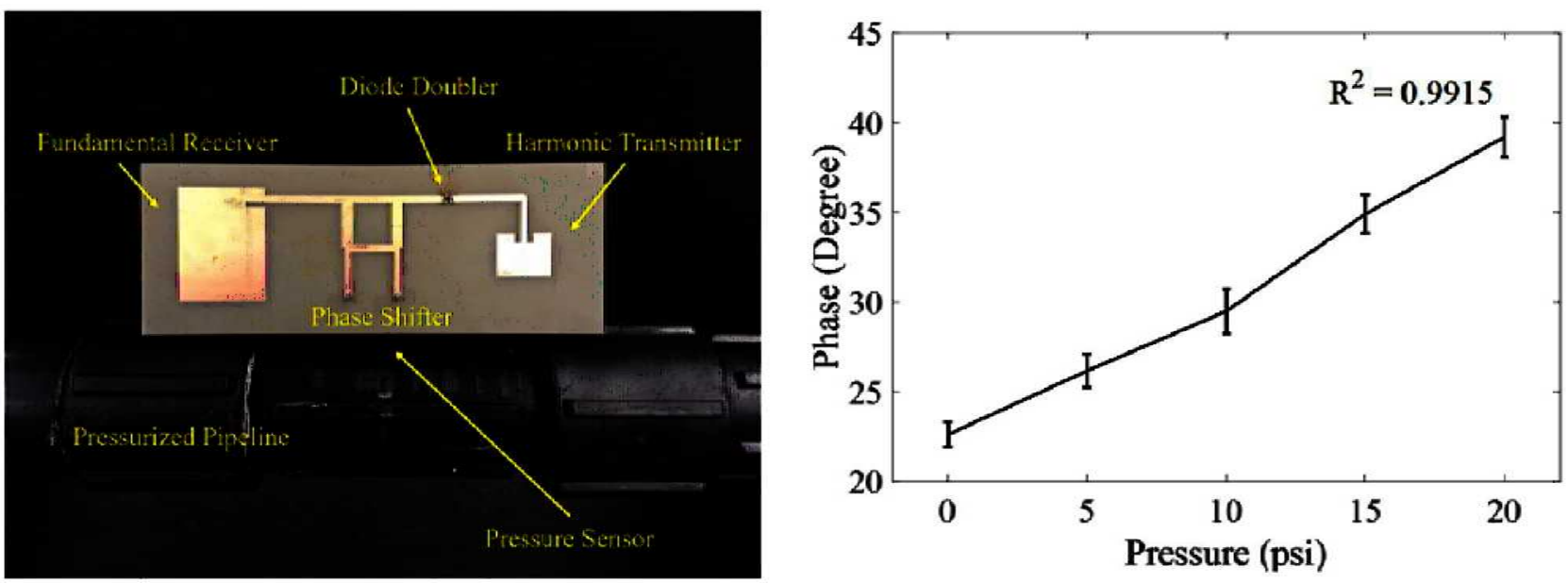

Figure 4. (Left) Pressurized pipeline setup with a harmonic RF sensor tag for real-time wireless pressure monitoring. (Right) Wirelessly measured phase change due to applied pressure.

A low-cost, 3D-printed pressure sensor and passive wireless sensing mechanism worked for monitoring the structural integrity of the gas pipelines, as shown in Figures 3 and 4 . The developed

harmonic pressure sensing system has good sensitivity (1) to reactive impedance, better linearity $\left(\mathrm{R}^{2}=\right.$

0.9915), a higher SNR, lower modulation losses, and a longer communication range. Harmonic communication eliminates self-jamming, reduces multi-path interference, and doubles the measurement sensitivity (phase change) due to applied pressure. The developed long-range wireless sensing platform enables continuous, safe, reliable, real-time monitoring of the pipeline and provides a cost-efficient solution for energy production plant operations.

Second topology [11]: A power-efficient, passive, analog-frequency-modulation-based RF system that enables real-time communication via multiple sensor elements for internet-of-things (IoT)-based smart applications. The designed battery-less RF tag with integrated sensors uses an onboard ultra-low- 
power oscillator to generate a single-frequency modulation signal unique to each sensor element. The generated single-tone frequency is modulated to the backscattered RF signal, which is immune to the interference in the propagating environment. The power required for continuous operation of the sensing oscillator is $30 \mu \mathrm{W}$, harvested from the received RF signal. The designed multi-sensor RF tag has a sensitivity of $-6 \mathrm{dBm}$ and can integrate up to six different sensors within the available single-channel RFID bandwidth of $300 \mathrm{kHz}$. A wireless communication distance of $10 \mathrm{ft}$ is achieved via the low power consumption of the multi-sensor RF tag. A passive RF tag with two example sensing elements - temperature and luminescence-was demonstrated, as shown in Figure 5. The temperature change from 25 to $60^{\circ} \mathrm{C}$ and the luminescence change from 0 to 20 foot-candle (lumen $/ \mathrm{ft}^{2}$ ) is represented by a $75-100 \mathrm{kHz}$ and a $125-150 \mathrm{kHz}$ frequency range, respectively.

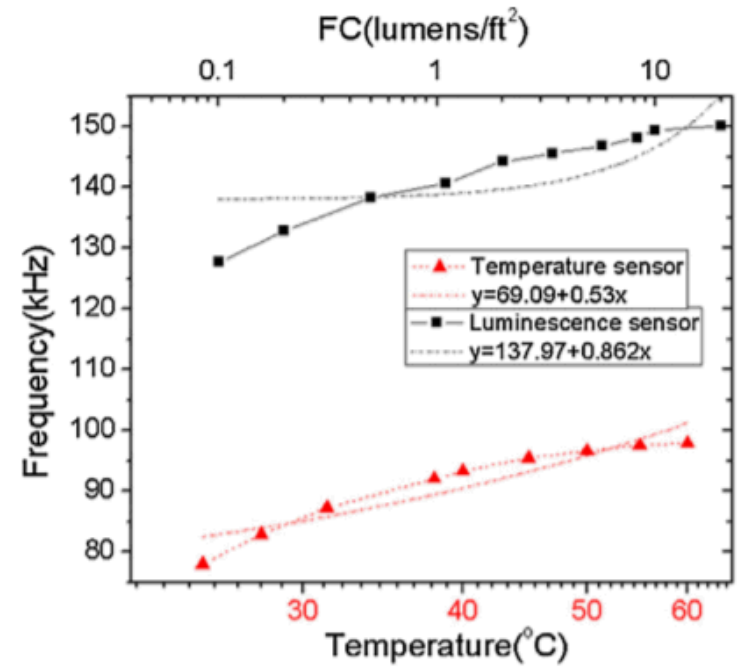

(A)

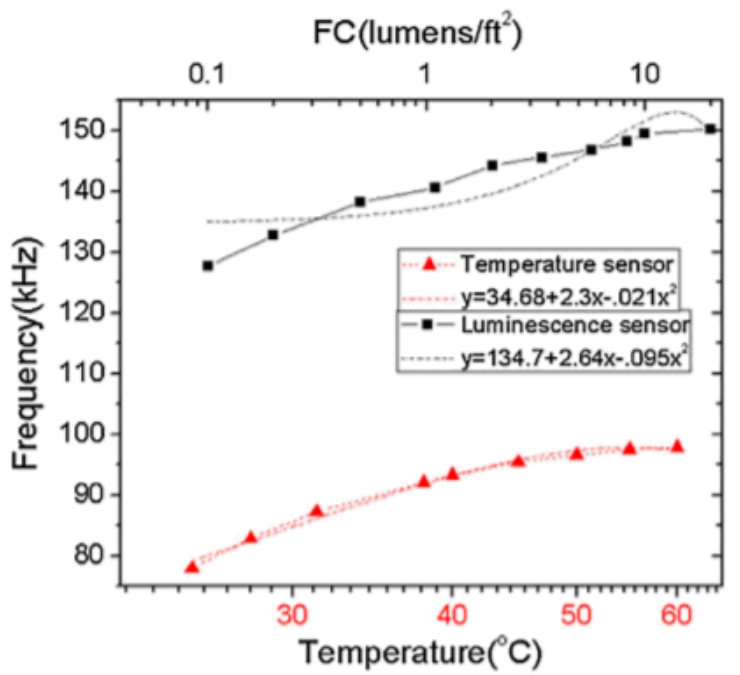

(B)

Figure 5. Frequency change of temperature and luminescence sensors with (A) linear fit and (B) secondorder polynomial fit.

The temperature and luminescence sensors reflect back the information signal in the form of modulated sideband frequencies. The received frequencies for the temperature and luminescence sensors are, respectively, $82.34 \mathrm{kHz}$ and $149.59 \mathrm{kHz}$, which correspond to $27.8^{\circ} \mathrm{C}$ and $13 \mathrm{fc}$. The temperature and lighting conditions of the experimenting lab are verified using a commercial temperature- and lightmeasuring instrument from URCERI. The frequency spectrum of the received demodulated signal is shown in Figure 6. The frequency signal (sensor data) peaks are clearly visible; the frequency modulation of sensor data is immune to any multi-path interference or noise in the propagation. The time domain representation of the demodulated RF signal is shown in Figure 6, where the information signal from the first and second sensors repeat over a time period of approximately $160 \mathrm{~s}$. The temperature and luminescence signals with a $82.34 \mathrm{kHz}$ and a $149.59 \mathrm{kHz}$ frequency, respectively, are shown in the zoomed-in section. The demodulated signal is amplified using a 40-dB low-frequency amplifier before acquiring the data using an oscilloscope. The sensors used in the system are analog in nature. Hence, their minimum resolution depends on the measurement instrument used at the interrogator. For a standard oscilloscope with $1 \%$ measurement accuracy for bandwidth up to $200 \mathrm{kHz}$, the minimum resolution is $2.6^{\circ} \mathrm{C}$ and $1.2 \mathrm{fc}$ for the temperature sensor and luminescence sensor, respectively. 

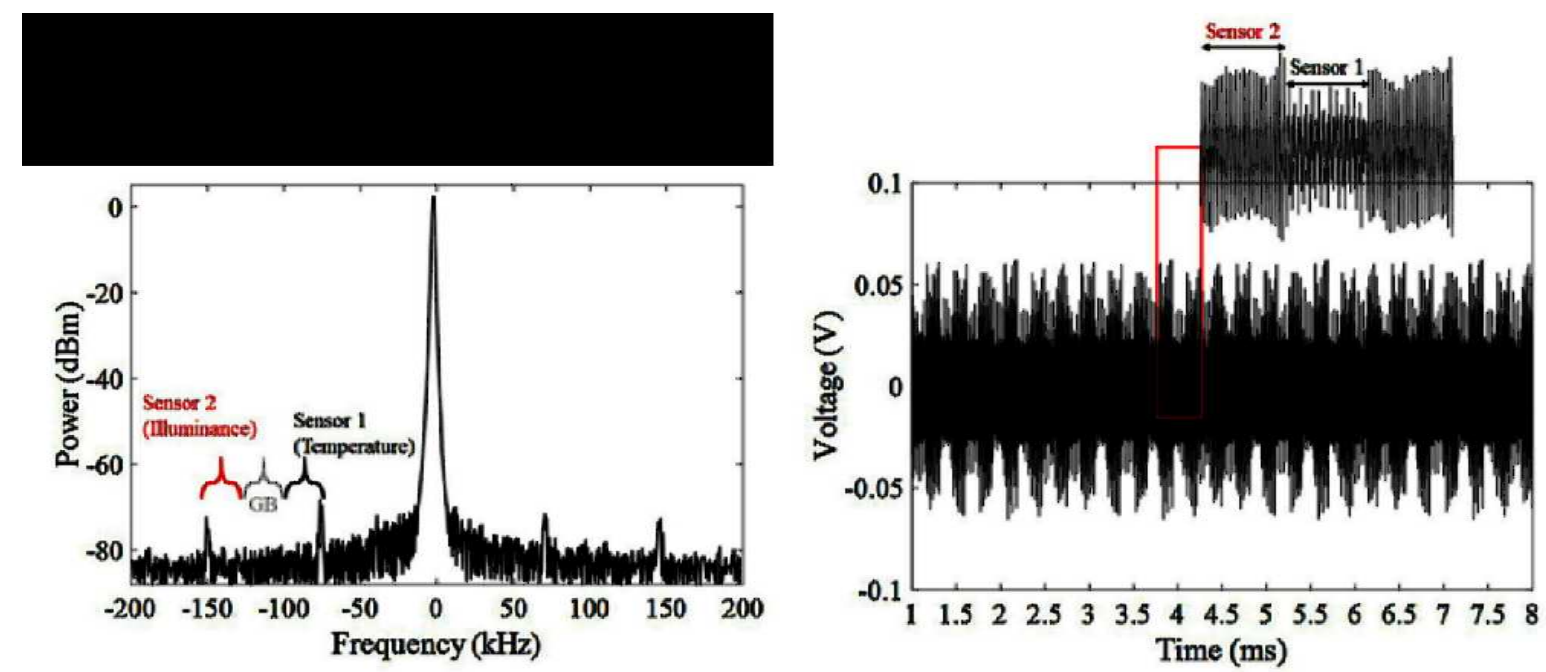

Figure 6. (Left) Frequency spectrum of the demodulated sensor data. (Right) Wireless demonstration with 9-dBm transmitted power at $908 \mathrm{MHz}$, showing received sensor information in a time domain with a switching frequency of $6 \mathrm{kHz}(\sim 160 \mu \mathrm{s})$.

Both wireless communication topologies are composed of powered interrogators and battery-less RF tags with integrated sensors. The interrogators can receive information from the sensing modules and relay it to the control room or multiple base stations. By collaborating with industry and within INL, communication to the base stations can be securely encrypted to prevent eavesdropping and cyberterrorism. The designed automated wireless monitoring network featuring passive integrated sensors will be scalable, reliable, and optimized for nuclear processes and assets.

Microfabrication techniques can be applied for the sensing nodes, which consist of multi-sensing elements with small footprints. For enhanced durability and reliability, the sensors and circuits are designed to be passive, thereby minimizing their need for shielding and protection in nuclear environments and other hazardous conditions. A typical sensing node will consist of the combination of sensing elements required for measuring various parameters salient to the operation of the reactor or the experiments conducted within. Sensors in different configurations can be developed to measure these salient parameters. The specific objectives of this passive communication architecture are as follows:

(1) To improve state-of-the-art nuclear monitoring sensors integrated with passive wireless communication systems

(2) To provide a robust, battery-less passive antenna system for monitoring and communication

(3) To enable simple, effective installation of the wireless measurement system.

The battery-less RF tags with single- or multi-parameter sensors have lifetimes that span decades. Since the sensors do not have batteries or any other active circuitry, they can withstand high temperatures and radiation in nuclear environments. Monitoring of certain parameters quantities in nuclear facilities - such as radiation, strain, pressure, and temperature - can be accomplished on a single microchip with temperature compensation strategies. All parameters can be sensed at a single location by implementing the sensor nodes onto a single chip. Such integrated passive-sensing RF tags for long-range communication can be strategically designed and developed for different nuclear applications.

The proposed concepts and feasibilities discussed in this section have been demonstrated in the literature $[1-2,6-7,11-12]$. 


\section{RFID SENSOR RUGGEDIZATION FOR HARSH ENVIRONMENTS}

The ability to take measurements within a developmental fuel system while the fuel undergoes testing and irradiation will considerably shorten the design period while strengthening our understanding of fuel performance under irradiation. Previous research demonstrated that PDCs possess unique thermal and mechanical properties that make them particularly suitable for harsh environments as RFID sensors. These include: (1) unique, tunable semiconducting behavior for temperature measurements, (2) high piezo-resistivity for pressure measurements, (3) excellent thermo-mechanical properties (in up to $1500^{\circ} \mathrm{C}$ ) for high-temperature survivability, (4) probable high radiation resistance for irradiation survivability, and (5) micro-fabrication capability to ensure mass production of the proposed sensor suite at low cost. The detailed material properties of PDCs as sensing materials are described in Section 4.3, while Section 4.4 provides the development path to enable the design and building of an RFID sensor based on the unique properties of PDC materials.

The RFID sensor system can be applied in LWRs, high-temperature gas-cooled reactors, and other reactor concepts, as EM waves have been shown to propagate through water. Because temperature sensors are, in general, the most expedient to implement and the simplest to corroborate, an RFID temperature sensor will be the first type developed. If this development is successful, several other types of sensors (e.g., heat-flux, stress, and strain sensors) can then be designed for taking measurements inside the reactor core.

\subsection{Plan for Field-Deployable Sensor and Signal Transmission}

Taking measurements in fuel rods is difficult since the fuel is inside a metal cladding. If the design challenge for transmitting signals in and out of the fuel rod is successfully resolved, other nuclear applications will become possible. The design concepts for transmitting energy into the rod and the signal out of the rod will be discussed in this section and can be generalized to include other fuel formats such as plate fuel and Tri-structural ISOtropic (TRISO) pebbles. The schematic diagram of the LWR-2 fuel rodlet and capsule assembly for Advanced Test Reactor (ATR) irradiation is shown in Figure 7. The LWR-2 fuel rodlet will need to be modified for an RFID sensor to be installed within. An example implementation for a field-deployable sensor is shown in Figure 8. The schematic diagram of the LWR-2 fuel rodlet and capsule assembly for ATR irradiation shows the capsule containing the fuel rod within (far right), the fuel rod (center), and a zoomed-in schematic of the RFID sensor (far left). The micro-sensor hangs inside the fuel rod cladding via a feedthrough in the cap. The sensor suite will be designed using passive sensors and will be powered wirelessly by an external pulse source. 


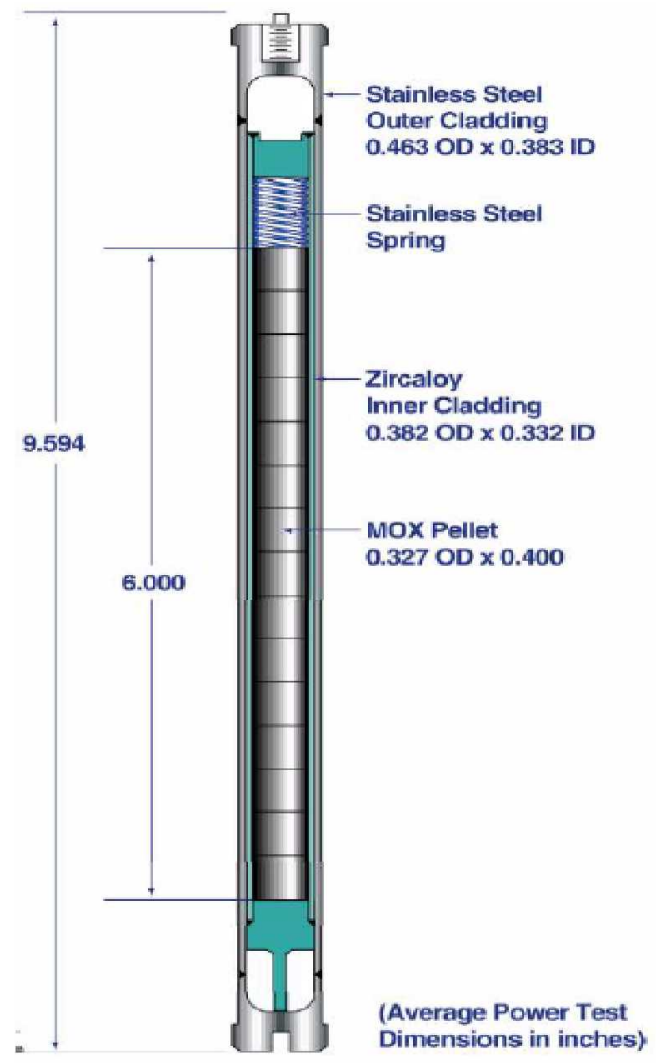

Figure 7. Schematic diagram of the LWR-2 fuel rodlet and capsule assembly for ATR irradiation. Dimensions are in inches. 


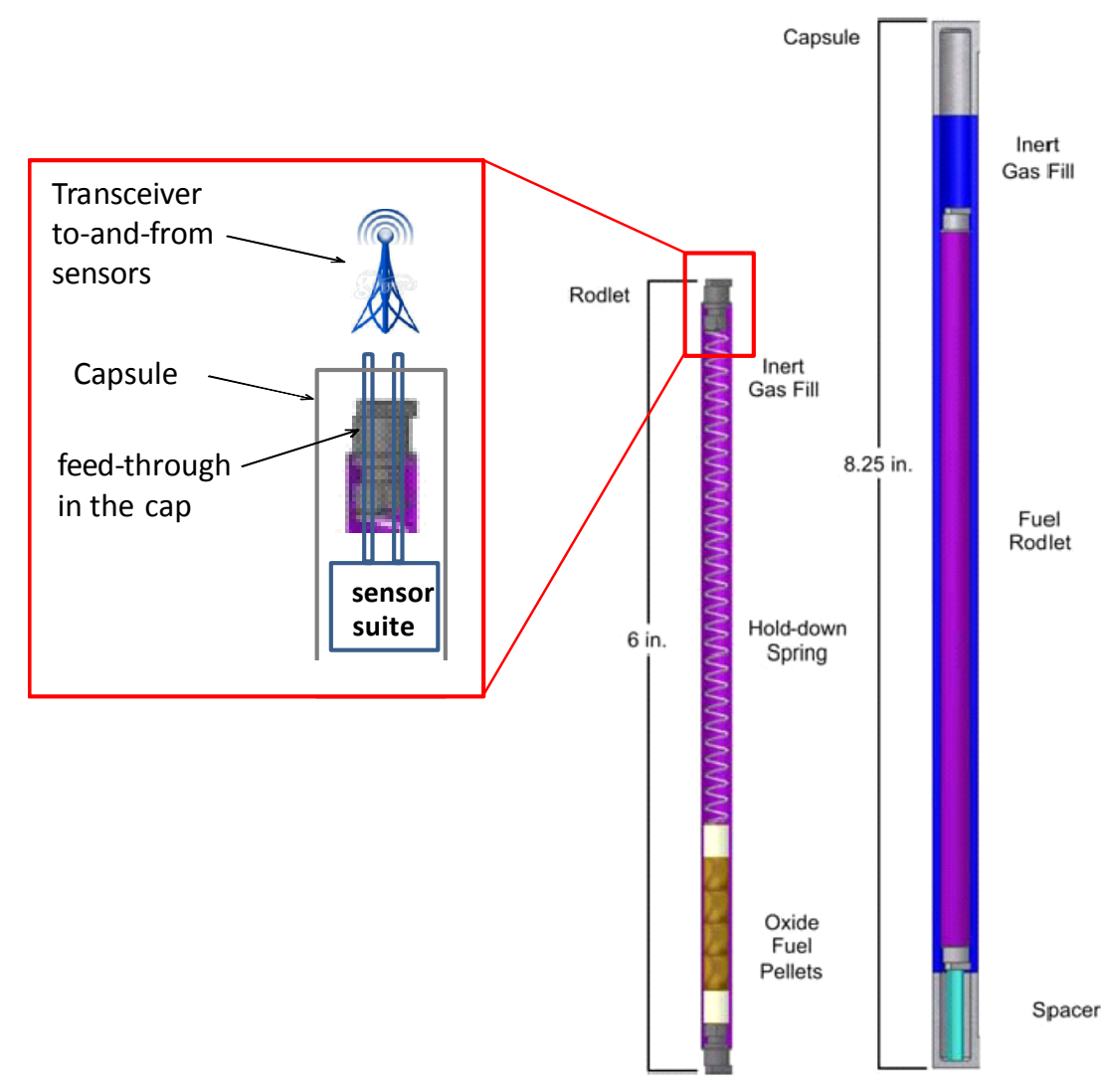

Figure 8. Typical oxide fuel drop-in capsule configuration (zoom-in: the sensor suite is "hung" inside the rodlet by an electrical feedthrough in the cap).

The wireless signal transfer is based on RFID technology that has been in use for decades. Figure 9 shows a schematic diagram of the proposed sensor concept. The RF tags are comprised of commercially available technologies and generally consist of three parts: transceiver, micro-patch antenna, and resonant circuit. The sensing system consists of a wireless transmitter/receiver. The antenna portion of the transceiver resides just inside the reactor primary containment vessel in a relatively low-radiation area. An RF signal will be transmitted to a high-temperature micro-patch antenna. Inside the fuel rod will be a PDC sensor connected to the micro-patch antenna feedthrough. Although use of wires in harsh environments is not desired, the wiring and installation of the PDC sensor into the fuel rod/capsule will be done in an instrument fabrication environment. 


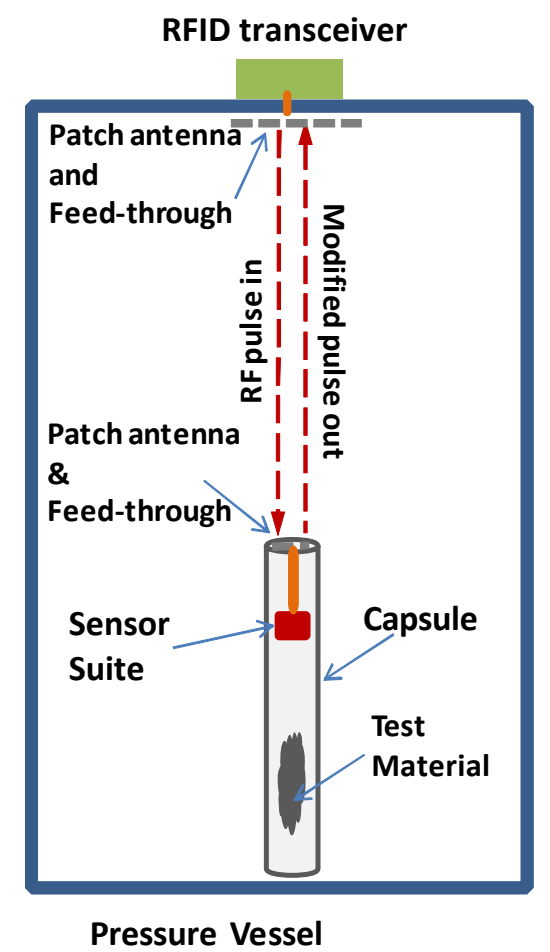

Figure 9. Schematic diagram showing the proposed sensor-transmitter system.

Recently, several methods were proposed and demonstrated for propagating the RF signal through water and/or other liquids in order to sense physical parameters (e.g., pressure and temperature) at a distance of up to $30 \mathrm{~m}$ (exceeding reactor deployment requirements), using various transmission frequencies ranging from $100 \mathrm{kHz}$ to $20 \mathrm{GHz}$ [13-19]. Previous results demonstrated the communication of temperature data using a commercially available active device, acquiring near real-time data at a sustainable rate of at least once per minute. In another proof-of-principle experiment, a commercial RFID development kit was used to test the signal reception in a water tank. With the transmitter located outside the tank and the receiver in the water, signal transmission through the water was not degraded in comparison to baseline air transmissions; the effective stand-off distance actually increased when transmitted through water. Therefore, the reliability of transmission through water via the RFID tag is equal to or greater than that of air-to-air transmission for this particular configuration.

The basic foundation of an RFID sensor is the LCR (inductor, capacitor, resistor) resonant circuit. The resonant frequency of the sensor changes in accordance with the change in the resistance of the resistor. As described in Section 4.4, the electrical resistance of PDCs changes with both the surrounding temperature and pressure, in turn changing the resonant frequency of the LCR circuit. This is the sensing mechanism behind the sensor suite with wireless signal transmission. During implementation, the transmitted RF signal will cause the PDC sensor to resonate at a center frequency based on temperature (or pressure). The resonant sensor re-emits a modified signal broadcasted by the micro-patch antenna for reception by the transceiver and data acquisition system.

\subsection{Design Objective}

The objective is to develop a novel micro-sensor suite for in-situ and real-time monitoring (with a sampling frequency of at least $1 \mathrm{~Hz}$ ) of temperature and pressure inside nuclear reactor fuel rod cladding. 
During preliminary laboratory experimentation [20], the measurement spanned 300-950 for 10 hours,

with no measurable performance degradation and a maximum error falling within 3. Compared to other

sensor technologies, the proposed system has the following advantages:

- Because made of PDCs, the sensor will survive in harsh reactor environments.

- The sensor dimensions can be made small enough $(\sim \mathrm{mm})$ to fit in the reactor core regardless of current space limitations.

- The measured temperature signal will be sent wirelessly out of the experiment capsule or the core, eliminating the need for connection cables and thus enhancing the reliability of the sensor system.

\subsection{Properties of Polymer-Derived Ceramics}

PDCs are a new class of high-temperature, multifunctional materials synthesized via thermal decomposition of polymeric precursors. The synthesis processing includes the following basic steps (Figure 10) [21, 22]: (1) synthesize/modify polymer precursors (SiCN), (3) shape and cross-link the precursor to infusible polymer components/devices, and (3) pyrolyze the polymer components/devices into ceramics through pyrolysis. The resultant materials will predominantly be amorphous ceramic, consisting of silicon, carbon, and nitrogen [23]. Other elements such as aluminum can also be incorporated into the network to tailor and improve the material properties [20, 24].

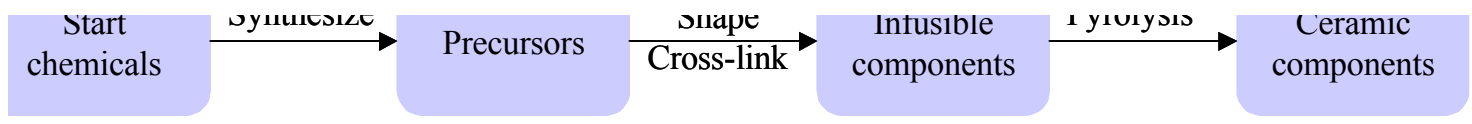

Figure 10. Processing steps for synthesizing polymer-derived ceramics [21, 22].

PDCs possess unique and superior properties that make them particularly suitable for the proposed micro-sensor suite in regard to in-situ and real-time temperature/pressure measurements in nuclear reactors. These properties include:

- Unique, tunable semiconducting behavior for temperature measurements

- High piezoresistivity for pressure measurements

- Excellent thermo-mechanical properties (at up to $1500^{\circ} \mathrm{C}$ ) for high-temperature survivability

- Probable high radiation resistance for irradiation survivability

- Micro-fabrication capability to ensure low-cost mass production of the proposed sensor suite.

\subsubsection{Unique and tunable semiconducting behavior for temperature measurement}

Recently, it was demonstrated that PDCs exhibit a stable semiconducting behavior at up to $1600^{\circ} \mathrm{C}$ [25-27], much higher than what other wide band gap semiconducting materials (such as $\mathrm{SiC}$ ) are capable of. Figure 11 shows typical PDC temperature-dependent electrical conductivity. The curves exhibit three 
regions, suggesting that the material exhibits typical amorphous semiconductor behavior. Figure 11 also illustrates that PDC electrical conductivity is perfectly repeatable during heating and cooling cycles, so sensors made from these materials can be repeatedly used in high temperatures. The thermal coefficient of resistivity, $k=\left(d R / R_{o}\right) / d T$ (where $\mathrm{R}$ is the resistance of the sample and $\mathrm{T}$ is the temperature), of this material is $>10^{-3}$, higher than for Pt, which has the highest thermal coefficient of resistivity among all currently used high-temperature sensing materials. This means that the temperature sensors can utilize the semiconducting behavior of PDCs, maintain high measuring accuracy, and survive in high temperatures of up to $1500^{\circ} \mathrm{C}$.

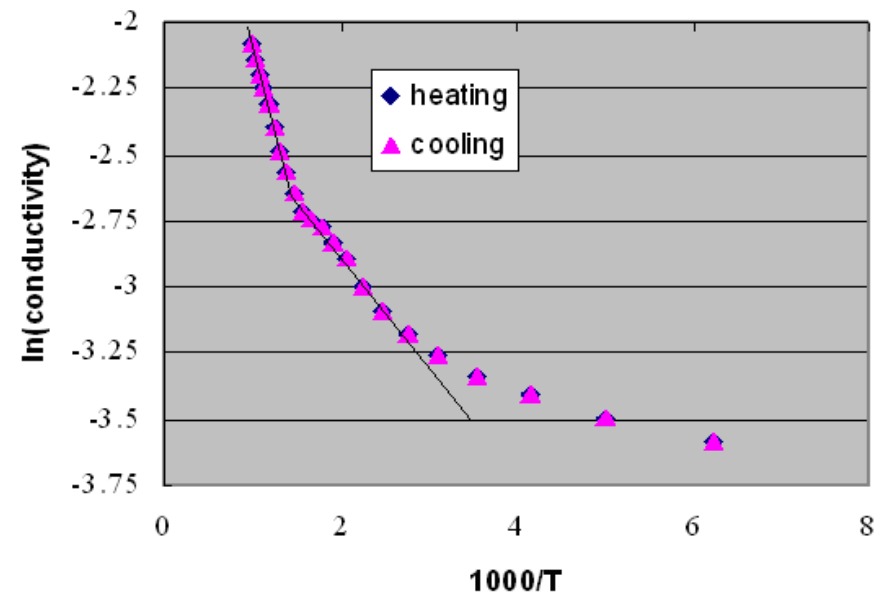

Figure 11. Electrical conductivity of a PDC as a function of temperature.

In addition, it was demonstrated that the electrical properties of PDCs strongly depend on the concentration of the free carbon phase and $\mathrm{C}$-dangling bond. By changing the concentration of the free carbon phase and C-dangling bond, the electrical conductivity of PDCs can be varied over a large range. Figure 12 shows the resistivity of PDCs as a function of composition. It is seen that the resistivity of PDCs can be varied by more than four orders of magnitude by simply varying the concentration of the modifiers. The capability to vary electrical conductivity over a large range is particularly important for the proposed applications, since the entire sensor can be fabricated from similar materials with different resistivities in order to avoid thermal mismatch/stress - the most critical issue in determining the reliability and lifetime of a sensor used in high temperatures. 


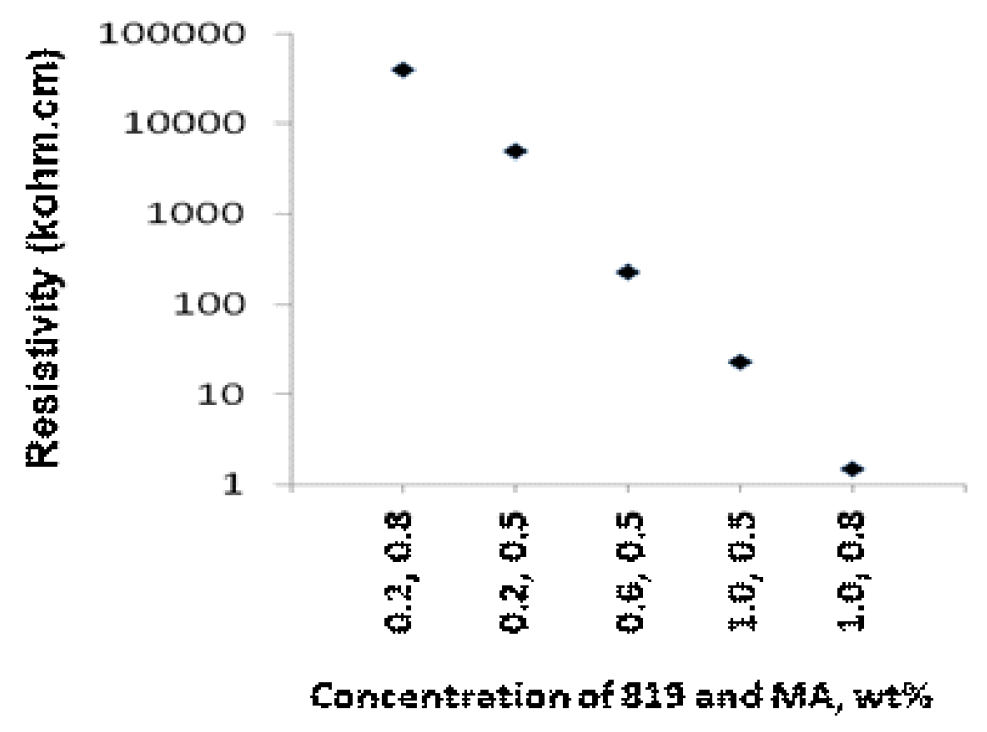

Figure 12. Electrical conductivity of PDCs as a function of composition.

\subsubsection{High piezoresistivity for pressure measurement}

PDCs also exhibit remarkably high piezoresistivity. Figure 13 shows the electrical resistance of a PDC material as a function of uniaxial compressive stress. It is observed that the resistance of the sample decreases in accordance with the applied stress. The resistance's dependence on stress is highly repeatable during loading/unloading cycles, in which changes are caused by elastic deformation. The strain gauge factors $k$ (defined as $k=\left(\Delta R / R_{o}\right) /\left(\Delta L / L_{o}\right)$, where $\Delta R$ is the change in resistance, $R_{o}$ is the original resistance [ohm], $\varepsilon=\Delta L / L_{o}$ is the strain, and $L_{o}$ is the original length [in meters]) are calculated from the curves. The range of the $k$ values is 1000-4000- significantly higher than that of $\mathrm{SiC}(k \sim$ 20-30). Therefore, pressure sensors made from PDCs will have much higher sensitivity and a wider measuring range. Since PDCs are amorphous semiconductors, the PDC gauge factor should have a weak temperature dependence $\left(\propto \mathrm{T}^{1 / 4}\right)$. As a result, pressure sensors made from PDCs are expected to have high signal gain at elevated temperatures. In contrast, the $k$ of crystalline materials such as $\mathrm{SiC}$ decreases significantly with temperature, leading to lower signal gain at elevated temperatures.

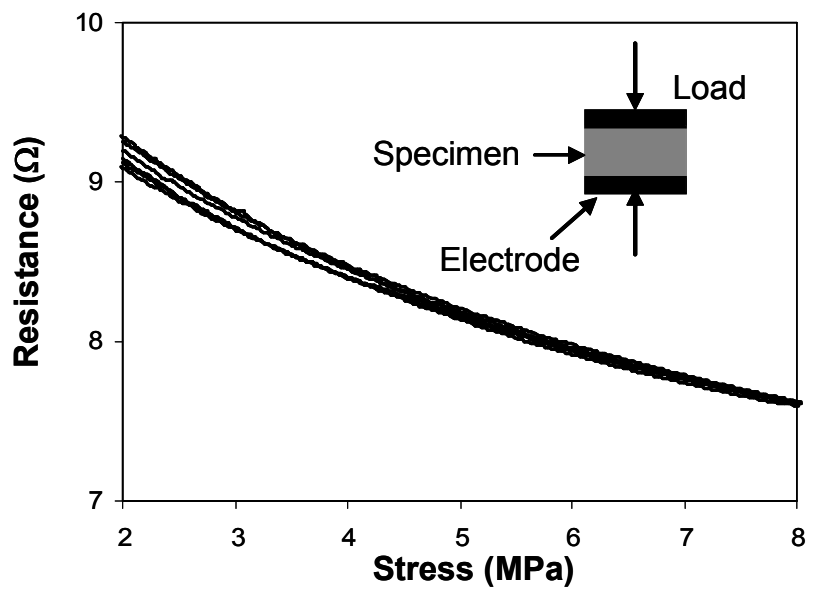

Figure 13. Electrical resistance as a function of applied stress [28]. 


\subsubsection{Excellent thermo-mechanical properties at up to $1500^{\circ} \mathrm{C}$ for high- temperature survivability}

It was demonstrated that PDCs exhibit excellent thermo-mechanical properties at up to $1500^{\circ} \mathrm{C}$, without any structural or composition degradations. The creep resistance of PDCs exceeds that of polycrystalline $\mathrm{SiC}$ and $\mathrm{Si}_{3} \mathrm{~N}_{4}$. In particular, $\mathrm{PDCs}$ exhibit an anomalously high resistance to oxidation and hot corrosion (Figure 14). Consequently, we anticipate that PDC-based sensors can survive the corrosive, high-temperature environments found in nuclear reactors.
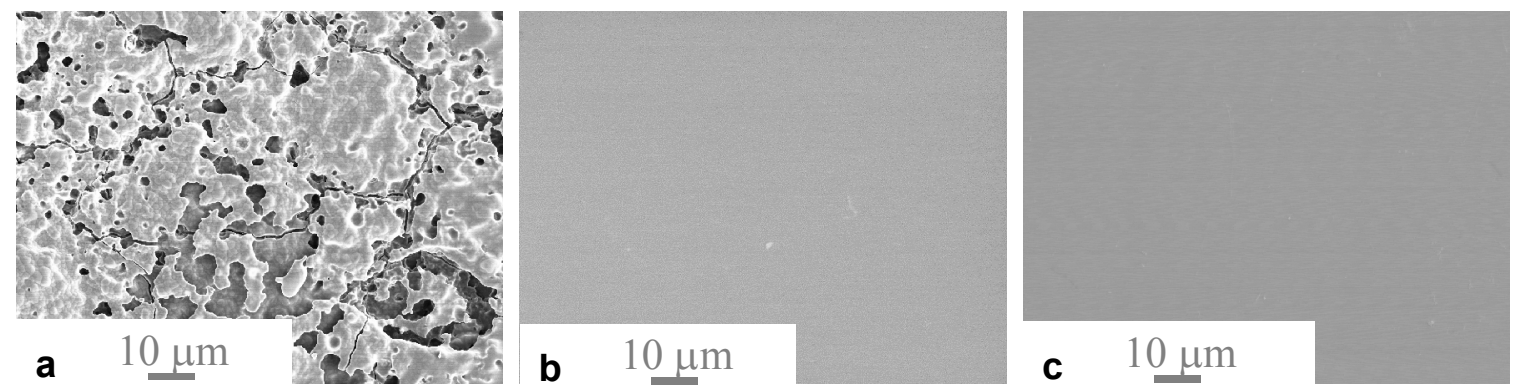

Figure 14. Scanning electron microscope images of the surfaces of (a) typical silicon-based ceramics after heat-treatment in water vapor and/or NaCl-containing environments, (b) PDCs after annealing at $1400^{\circ} \mathrm{C}$ for $300 \mathrm{hrs}$ in $50 \% \mathrm{H}_{2} \mathrm{O}-50 \% \mathrm{O}_{2}$, and (c) PDCs after annealing at $1200^{\circ} \mathrm{C}$ for $50 \mathrm{hrs}$ in a NaCl-containing environment [29].

\subsubsection{Probable high radiation resistance for irradiation survivability}

While PDCs' radiation damage behavior has not yet been studied, their known behavior suggests they should be much more radiation-resistant than $\mathrm{SiC}$ and $\mathrm{Si}_{3} \mathrm{~N}_{4}$ ceramics. It is well-known that the root cause of radiation damage is the continuous generation of vacancies and interstitials in atomic displacement cascades and defect accumulation. Crystallinity of the material is thus an important criterion in causing radiation damage to occur. PDCs are mostly non-crystalline, making them damage-resistant. The unique structure of PDCs can effectively promote defect recombination to mitigate radiation damage.

In our prior research, PDC (SiAlCN) ceramics were prepared and tested under irradiation. The irradiation test was performed by Dr. Jay Kunze and his team at Idaho State University. The irradiation lasted three hours at 4 watts. The sample received a gamma dose of about $240 \mathrm{~Gy}$, equal to 24,000 rads of gamma radiation (or 24,000 rads equivalent man). The sample saw a neutron dose-essentially all from fast neutrons - of 120,000 rads equivalent man. According to the optical images, the PDC ceramic sample maintained its integrity after the irradiation test.

\subsubsection{Micro-fabrication capability to ensure low-cost mass production of the proposed sensor suite}

Another attractive property of PDCs is that they can easily be micro-machined into miniaturized components with complex features/structures in organic form, then converted into ceramics via pyrolysis, thus drastically reducing the manufacturing costs for mass production. A micro-mechanical machining technique was recently developed (in a lab) for making ceramic micro-electro-mechanical systems from PDCs [30-31].

\subsection{Research Tasks}

To achieve the aforementioned objectives, the following development tasks are necessary. 


\subsubsection{Task 1: Developing PDC material with desired electrical properties for accurate sensor measurement}

In this task, the compositions and structures of PDC materials will be systemically tailored to achieve the desired sensitivity and measurement accuracy for the designed sensor suite. PDCs consist of disordered graphite nanoclusters and a heterogeneous amorphous matrix. They are x-ray amorphous but exhibit some level of order and heterogeneity at the nanoscale. The size and amount of the graphite nanoclusters (referred to as free carbon), as well as the local composition, significantly affect the electrical properties.

Previous studies demonstrated that PDCs' electrical conduction strongly depends on the concentration of the free carbon phase. When the concentration rises above a critical level (depending on the size of the free carbon), the materials exhibit a tunneling-percolation effect, thus achieving significantly high piezoresistivity. On the other hand, when the concentration is below the critical value, the materials exhibit a semiconducting behavior that depends on the matrix composition.

\subsubsection{Task 2: Designing and fabricating the sensor suite for simultaneous temperature and pressure measurements}

Temperature measurement: Previous work [4, 5, 20, 21, 31-34] has primarily focused on using PDCs as high-temperature sensing material. Figure 15 shows a sample PDC sensor fabricated in the lab, along with the reference thermocouple. As described in Section 4.3.1, PDC materials exhibit temperaturedependent electrical conductivity. The thermal coefficient of resistivity is also higher than for Pt, affording accurate temperature measurement. During actual measurements, the PDC temperature sensor can be simplified as a potentiometer whose resistance changes according to the ambient temperature. A schematic diagram of the temperature sensor is given in Figure 16.

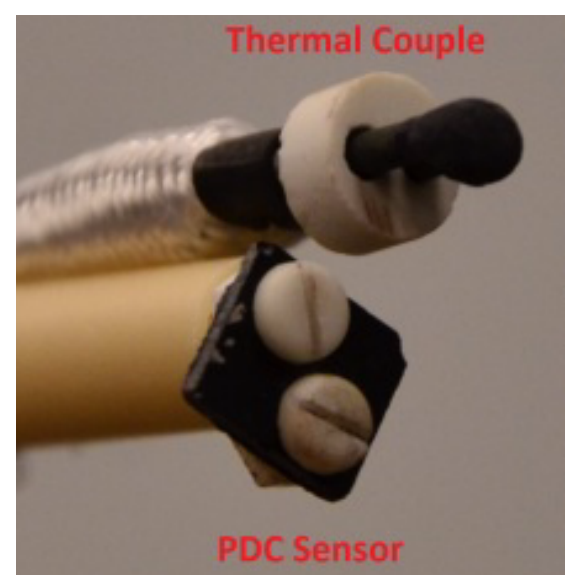

Figure 15. PDC sensors and reference thermocouple. 


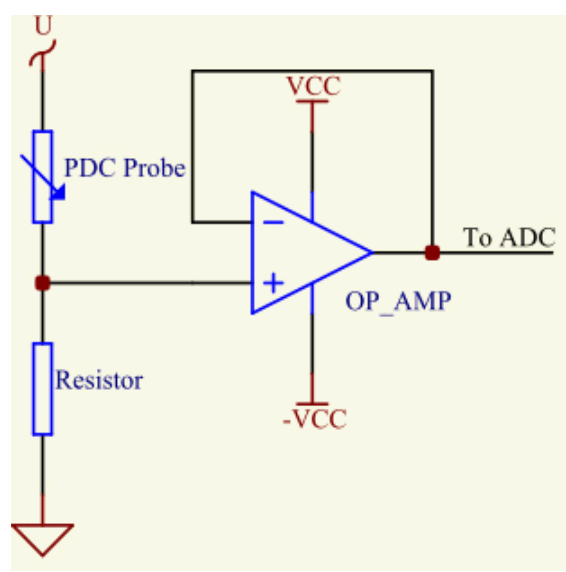

Figure 16. Signal conditioning circuit for a temperature sensor.

Repeatability tests for temperature measurement: Preliminary experiments demonstrated very consistent thermal-resistance responses by the PDC sensor to temperatures as high as 950 The repeatability of the material's thermal-resistance behaviors ensures accurate temperature measurements, and a Wheatstone bridge was designed to convert the resistance change into a voltage signal. The PDC sensor was then calibrated using the measurement from a reference thermocouple. In a repeatability test

in which the temperature was changed from 650 to 830 and cycled six times for a total of 10 hours,

results showed a maximum measurement error of 3 .

Sensitivity adjustment for higher-temperature measurements: By adjusting the Wheatstone bridge, the resolution of the PDC sensor can be shifted toward higher temperatures without sacrificing the SNR. This

is guaranteed by (1) the high resistance sensitivity of the PDC material (2.86 between 950and 1050)

and the fact that (2) the characterization algorithm works in the frequency domain, thereby avoiding the peak of the noise spectrum.

\subsubsection{Simultaneous temperature and pressure measurements}

The harsh environments of nuclear reactors may distort the sensor measurement signals, since PDCs respond to both temperature and pressure variations. As such, sensor data fusion will be performed to foster more accurate measurements. To extract accurate information, PDC sensors of different structures and compositions will be designed, fabricated, and characterized in a pressurized high-temperature container simulating the actual reactors. In the actual application, the temperature sensor will take measurements in the same environment as the pressure sensor for post-acquisition removal of temperature coefficients. 


\subsubsection{Signal conditioning system and wireless transmission}

As mentioned, the sensor suite will be designed to be passive and will be powered wirelessly by an outside source. During temperature and pressure measurements, the electrical resistance of the PDC sensor suite changes, altering the resonant frequency of the LCR circuit. All data-processing will be done outside the reactor vessel, as shown in Figure 9. Currently, this technology is at TRL-4.

\section{ELECTROMAGNETIC COUPLING}

Although RFID sensor technology potentially affords large standoff distances from the transceiver antenna, it has the disadvantage of requiring electrical feedthroughs to transfer information in and out of closed metal containers. When sensors are contained in closed metal structures that experience harsh conditions such as high temperatures, pressure, and radiation, through-wall communication of data and power enable sensor implementation without the need for penetrations. The resulting lack of sensor penetrations enhances sensor reliability and application safety.

EM coupling is now used to transmit signals and power through metal walls [35-27]. Three EMcoupling-based techniques were considered in the literature [35]:

1. Capacitive coupling

2. Magnetic resonance coupling

3. Inductive coupling.

It is reportedly impractical to use the capacitively coupled power transfer (CCPT) method to transfer power or data through a sealed metallic structure and to the sensors inside [35]. Although there are reports of power transfer though plates, no such reports demonstrate data transmission through metal walls (contiguous) using the CCPT method. Since there is no known remedy for this application issue, the CCPT technique is not discussed here further.

Literature demonstrating magnetic resonance coupling for through-metal-wall power transfer is scarce. A paper was found that discussed wireless power transmission into the interior of a closed metal box via magnetic resonance coupling [38]. The prototype system operated at a resonance frequency of 50 Hz. This low frequency was selected to reduce eddy current losses within the metal walls of the enclosure. It was demonstrated that $3 \mathrm{~W}$ of electric power was transmitted to light emitting diodes (LEDs) through a 1-mm-thick stainless-steel wall. The reported efficiency was approximately $40 \%$ over a transmission path of $12 \mathrm{~cm}$. It was also reported that $1.2 \mathrm{~W}$ of power was transmitted to LEDs by propagating through a metal pipe wall 5 -mm thick. The reported efficiency was $10 \%$. The reported efficiencies for this magnetic resonance technique are significantly better than for techniques using inductive coupling at the same frequency.

Magnetic resonance coupling is essentially the same as inductive coupling, except that magnetic resonance coupling uses a high-permeability core within the coils. The core significantly increases the efficiency of the coupling system. The addition of the core within the windings also increases the size and complexity of the communication system. Reducing the size of the core to decrease the footprint reduces the efficiency of the coupling system, and the harsh radiation environment (temperature and radiation) deleteriously affect the permeability of the core, driving the magnetic resonance coupling technique's efficiency closer to that of the inductive coupling technique. Thus, the robustness of the magnetic resonance coupling technique in a radiation environment is suspect. The magnetic resonance technique is better-suited for less harsh environments with significant space available.

Inductive coupling has been used to transmit data and power through thin metal barriers. It is probably the most common wireless data/power transmission method currently employed. It operates on a similar principal as that employed by electrical transformers: inducing magnetic field coupling via a pair of coils. A transformer generates an alternative magnetic field by having a current within the primary coil. 
The primary coil current creates current in the adjacent secondary coil via EM induction. In a transformer, the primary and secondary coils are tightly coupled to the core. As stated earlier, the inductive coupling system contains a detached primary and secondary coil pair. Inductive coupling was demonstrated to be useful for applications such as low-energy-transfer sensor applications [35]. Inductive coupling was demonstrated using a working prototype featuring low data rates and power transmitted through metal barriers to simultaneously power the sensor and communication electronics [39]. The demonstration produced a communication rate of greater than $100 \mathrm{bps}$ through a 20 -mm-thick stainless-steel plate.

Inductive coupling is a promising technology for use in radiation experiments. The inductive coupling system can be made from robust, passive electrical circuits. Both power and data can be inductively coupled through metallic walls [40]. However, there are two potential downsides to this technology for use in radiation experiments:

- The power necessary to drive the transmitter coil

- The standoff distance between the transmitter and the receiver coils.

The combination of these two potential issues means that the transmitter coil will require external powering; in turn, necessitating wired electrical connections. The next section discusses an inductivecoupling-based temperature-measurement system being implemented within a nuclear test reactor. This installation highlights the benefits and potential issues involved with inductive coupling technology.

Although EM coupling techniques show promise for use in radiation environments, EM coupling is only practical for use with specific metals and geometries. EM coupling can only be used with nonferromagnetic metals and thin walls. These limitations result from the Faraday shielding effect. EM coupling is only useful for data and power transmission at a low transmission rate $(\approx 100 \mathrm{~Hz})$ and in lowpermeability materials such as stainless steel, aluminum, titanium, etc.

\subsection{Inductive Coupling Implementation}

Section 4.3 discussed an inductive-based sensor designed and tested in the Massachusetts Institute of Technology Reactor (MITR) by Westinghouse [41]. It is estimated that this technology is at TRL-3-4. The sensor is based on a pulsed induction and receiver technique to transmit a pulsed signal from a source transmitter ideally located in an instrument thimble or within a nearby fuel rod. The induction pulse transmits energy through the fuel rod wall to the inductive sensor within. The inductive sensor is also a resonant circuit that will resonate and transmit a modified signal back to the receiver located near the transmitter. The inductive sensor can be designed to be sensitive to the following measurement parameters:

- Centerline fuel temperature

- Fuel rod internal pressure

- Fuel pellet stack elongation.

Changes in the inductance and capacitance, caused by a measurement parameter variation, will result in a shift in the resonant frequency, as demonstrated by Equation 1, where $\mathrm{L}$ is the inductance and $\mathrm{C}$ is the capacitance that comprise the resonant circuit. 


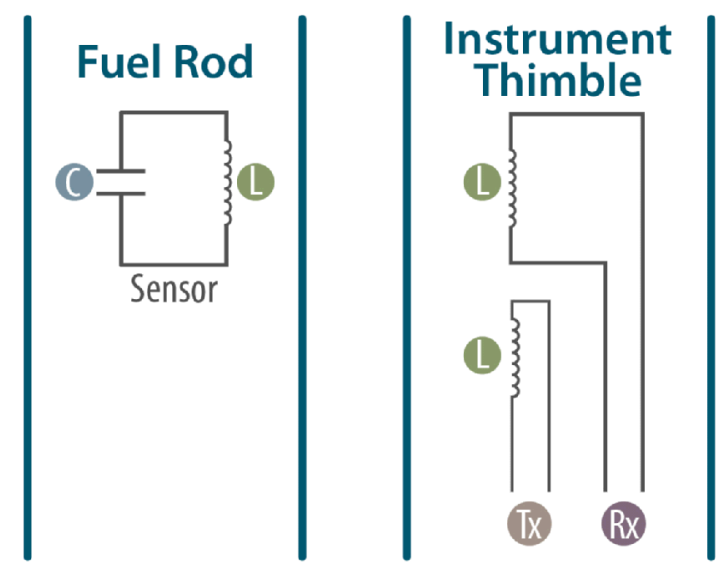

Figure 17. The inductive-sensor system design used in the MITR (redrawn from Carvajal et al.) [41].

The transmitter (Tx) coil is housed in the right-most ceramic capsule within Figure 17. The Tx continually pulses inductive energy through the fuel rod wall and into the sensor resonant circuit. The sensor resonant circuit modifies the resonant frequency of the re-radiated magnetic pulse based on the current values of $\mathrm{L}$ and $\mathrm{C}$. The transmitter must be switched off for the receiver ( $\mathrm{Rx}$ ) coil to pick up the returned signal. The fuel rod sensor/resonator is embodied by the resonant circuit housed in the ceramic capsule on the left in Figure 17. The Tx and Rx coils are shown in the ceramic capsule on the right, which represents the instrument thimble of a commercial LWR.

Testing of the inductive sensing system in the MITR was accomplished using ceramic capsules. The ceramic fuel capsules significantly increased the inductive transmission of the pulses for this inaugural test within the reactor. Inductive signals have been obtained through steel fuel cladding in the lab, as orally presented in [41] and discussed in [42].

The purpose of the inductive sensor test was to ascertain the performance of the sensor with respect to drift and robustness to temperature and radiation. The inductive senor was placed in a waterflow loop with the following characteristics: it was kept at $296^{\circ} \mathrm{C}, 10.3 \mathrm{MPa}$, and a waterflow rate of $18 \mathrm{~L} / \mathrm{min}$. The irradiation experienced by the sensor was estimated. When the reactor was set for $5.9 \mathrm{MW}$, the average neutron flux at the sensor location was $1.9 \times 1,014$ neutrons $/ \mathrm{cm} 2 / \mathrm{s}$ total and $8.3 \times 1,013$ neutrons $/ \mathrm{cm} 2 / \mathrm{s}$ for energies over $0.1 \mathrm{MeV}$. The gamma flux within the same location was estimated to be $2 \times 1,014$ gammas $/ \mathrm{cm} 2 / \mathrm{s}$.

Details of the experiment and the results thereof can be found in [41, 42]. The highlights of the results are as follows: the sensor system operated in the MITR autoclave loop functioned during exposure to an overall neutron fluence of $1.1 \times 1,021 \mathrm{n} / \mathrm{cm} 2(>0.1 \mathrm{MeV})$ and a cooling water temperature of approximately $300^{\circ} \mathrm{C}$. Amplitude variation and frequency changes occurred during operation, but the sensor system transmitted data throughout the testing.

The center frequency of the resonant inductive circuit drifted over a three-week period. This drift can be attributed to changes in sensor capacitance, since inductance measurements of the Tx and Rx inductors exhibited nominal variations in inductance during testing. Resonant frequency fluctuations during irradiation testing varied from 32 to $68 \mathrm{kHz}$. Data suggests that during the last 1.5 weeks, the drift was steadying at $68 \mathrm{~Hz}$. 
The signal amplitude was seen to diminish during the same three-week period. The signal loss is thought to result from a growth in the equivalent series impedance of the capacitor, or the inductor, or both. The impedance of the Tx and Rx coils grew in accordance with temperature and radiation during the start of the test but became stable after the initial rise. This indicates that the capacitor was the source of the long-term signal degradation. The total fluence seen by the sensor and transceiver in the MITR was compared to a nominal 40-year fluence within the plenum region of a pressurized-water reactor fuel rod. The equivalent timespan would be one to nine 18-month fuel cycles. The equivalent timespan heavily depends on the fuel rod's location in the pressurized-water reactor. MITR testing of the prototype inductive-sensing system successfully demonstrated that it can function in a nuclear reactor by wirelessly transmitting analog data. However, the frequency variation must be removed or compensated for before this technique can be used for measurement applications.

\subsection{Radio Frequency Modulator Implementation}

An RF-based communication system for use in a reactor core was developed by Westinghouse using radiation- and temperature-resistant electronics. Self-powered neutron detectors or thermocouples provide a measurement signal to a wireless transmitter containing vacuum micro-electronic (VME) circuits that broadcast the signals to a receiving antenna inside the reactor vessel. The signal from the antenna is routed to a data acquisition and processing system. The key technology enabling the radiation-tolerant transmission of signals is the VME-based amplitude modulation (AM) RF transmitter [41, 43].

Because traditional semiconductor circuits do not survive long in high-radiation fields, vacuum tube technology is utilized as the active elements within the reactor core. In general, passive components such as ceramic capacitors, quartz crystals, and wire wound resistors are naturally resistant to radiation. Radiation-hardened components are used to construct an RF oscillator operating at approximately 20 MHz that functions as a tank oscillator [44, 45]. The VME-based AM wireless transmitter that was developed can survive fast neutrons and gamma radiation at larger dose levels than commercial radiationhardened electronic components.

The radiation-hardened components are used to fabricate a capacitive oscillator with a shorting device comprising the active element in a relaxation oscillator that modulates the RF tank oscillator. Figure 18 is a high-level schematic of the RF transmission system. A tank circuit is comprised of a capacitor coupled by wires to an inductive coil. As the capacitor receives an electrical charge from a sensor, positive and negative charges build up on opposite ends of the insulating surface between the capacitor plates. Although opposite charges attract, these charges cannot move through the insulator but are forced to flow within the wires through the inductor coil. This movement of electrons electromagnetically charges the inductor. The electron movement between the capacitor and the inductor causes resonance in the tank circuit. As electrons move from the capacitor through the coil, EM energy in the capacitor is transferred to the inductor. Once the inductor contains more EM energy than the capacitor, energy is transferred back to the capacitor. The energy transfer restarts and repeats. Secondary elements used for EM transfer of the RF signal include enameled wire and NiZn ferrite material for an electrical transformer core.

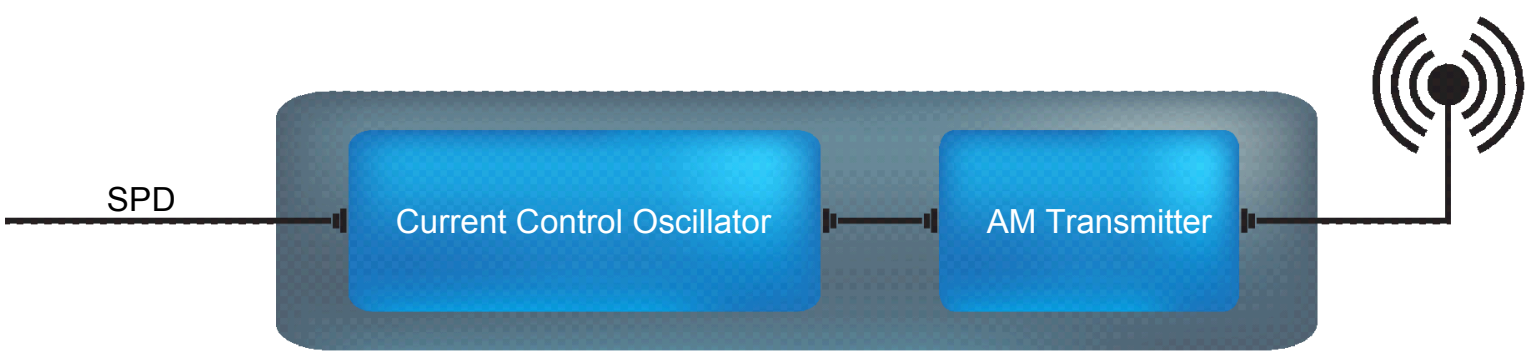

Figure 18. The self-powered detector signal is used to modulate an AM transmitter built from VME components and transmitted to a data acquisition system outside the reactor (redrawn from [44]). 
This wireless transmitter was demonstrated in the Breazeale Reactor at Pennsylvania State University. The Breazeale Reactor supplied a neutron flux of $1 \times 1,012 \mathrm{n} / \mathrm{cm} 2 / \mathrm{sec}$ and a total neutron fluence of $3 \times 1,018 \mathrm{n} / \mathrm{cm} 2$ to the transmitter system. Testing occurred over several weeks, with no component failures noted. Initial testing at the Breazeale Reactor showed that wireless transmission can successfully relay the modulated signal. However, changes in the reactor core setup over time, necessitated by normal reactor operations, caused intermittent signal reception. It was decided that subsequent tests would rely on a direct wired connection between the transmitter and the receiver. While this is disappointing, the technique of using the AM rate to encode reactor power was successfully demonstrated.

The resulting data [41] show that reactor power movements correlate to the modulation rate transmitted by the VME oscillator. The AM transmitter response is prompt and repeatable when the reactor transitions from 0 to $100 \%$ power. Step changes of $25 \%$ for powers of 100,75 , and $50 \%$ were closely tracked by the modulation rate. Changes in powers of less than $25 \%$ were not followed because the VME circuit cannot process a low Self-Powered Detector (SPD) current. For the Breazeale Reactor, the minimum detectable current would be close to $150 \mathrm{nA}$.

While the basic concept of AM encoding of reactor power was demonstrated using VME-based circuits within an active nuclear core, there are further technical challenges. The full vison of the transmission system was not implemented (see Figure 19). Self-powering of the transmitter (via SPDs), as envisioned in the patent [44], was not implemented. Active devices (transmitters) were powered by external power supplies, and the output from the SPD was wired into the transmitter after leaving the reactor pool. It is also interesting to note that the Breazeale testing used an AM transmitter and not the FM design described in the patent [43].

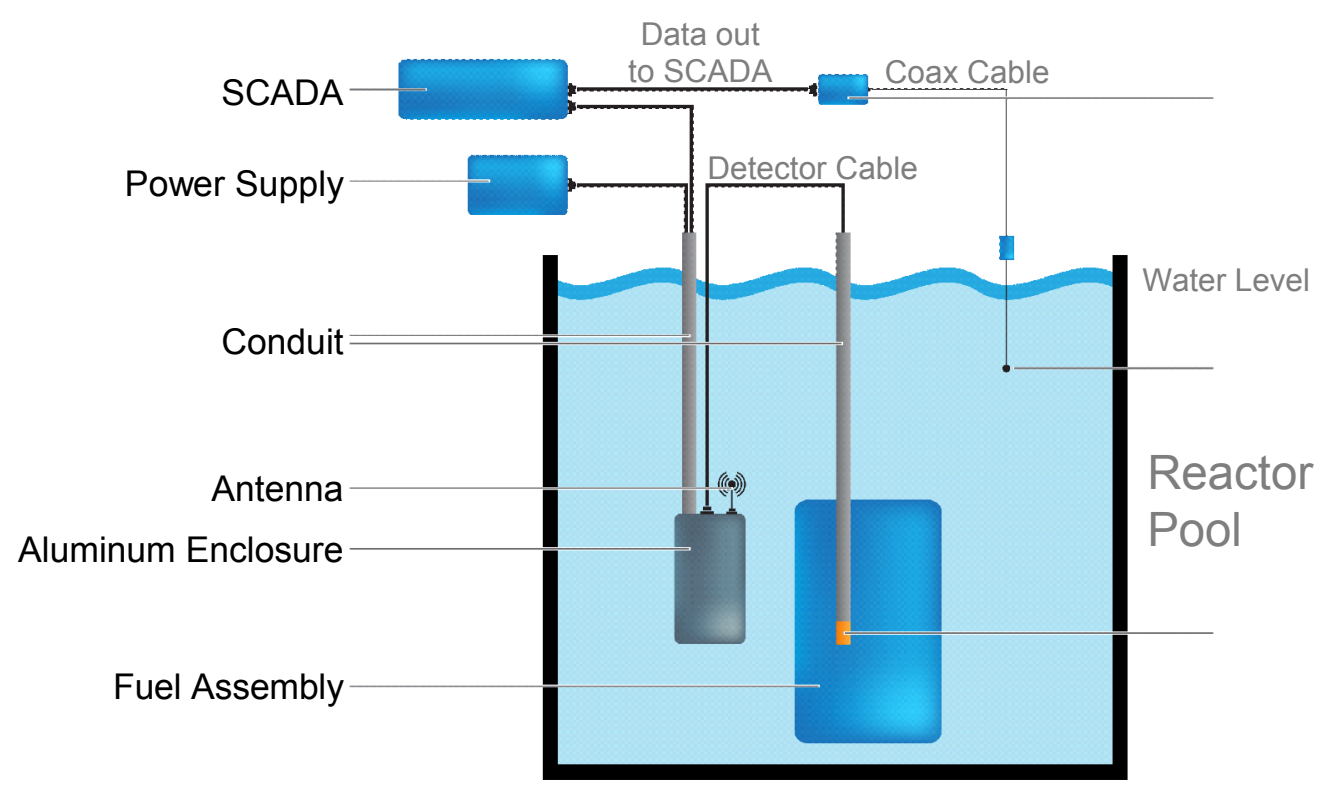

Figure 19. Experimental implementation of the wireless transmission system in the Breazeale Reactor, showing the external powering of the transmitters and the wired delivery of the SPD signal. The antennas were eventually replaced by cable connections due to intermittent signal transmission (redrawn from [41]).

At present, Westinghouse seems focused on developing their inductive technology. Westinghouse is working with INL to implement their inductive designs in the ATR for testing as part of the Accident Tolerant Fuel Program. It is believed that the reason for focusing on inductive technology is due to past experience with intermittent signal transmission and the need for an in-pile power supply. The power 
necessary to operate the VME transmitter broadcasting the RF signal is planned to be provided by a device that converts gamma radiation into electrical current [43]. The power source is to be co-located in the reactor along with the VME transmitter. The local power source will eliminate the necessity to deliver power from outside the reactor. However, the gamma power harvesting device remains a future work [41].

\section{WIRELESS SENSOR INSTALLATION RECOMMENDATIONS}

The previous sections of this report described how to generate signals within the reactor. This section suggests general approaches for transmitting the signals out of the reactor. Because current and advanced reactor designs are complex and extremely diverse, it is beyond the scope of this report to provide specific transmission designs. The sensing techniques discussed here focus on EM techniques, with conductive coolants such as molten salt or liquid metal providing significant challenges in regard to EM transmission. The possibility of multipath interference due to RF transmission is high, since dominant structural materials in reactors are metallic. The techniques discussed in Section 3 can be used to reduce the effects of multipath interference.

Despite the diversity of reactor types, the functional aspects of all reactors are similar: heat source and flowing coolant. The flowing coolant channels and orifices can provide EM transmission paths and access points in reactors. Due to structural shielding, not all fuel elements are likely to be accessible, but a significant number of them should be. The next subsection uses an LWR core as an illustrative example.

\subsection{LWR Installation Concepts}

In Section 4.1, a simplified field-deployable sensor and transmission concept was discussed, as depicted in Figure 9. A representative fuel assembly element provides access to the ends, as shown in Figure 20. The wireless transmission system would be easier to implement using the top cap of the fuel rod. The top of the reactor is generally the avenue for installing fuel and instrumentation. Thus, the transceiver could be installed at the top of the reactor and radiate down onto the tops of the fuel rods. Furthermore, the Fabrication of the micro-patch antenna feedthrough can be built into the cap of the fuel rod, simplifying the installation of the RFID sensor into the fuel rod.

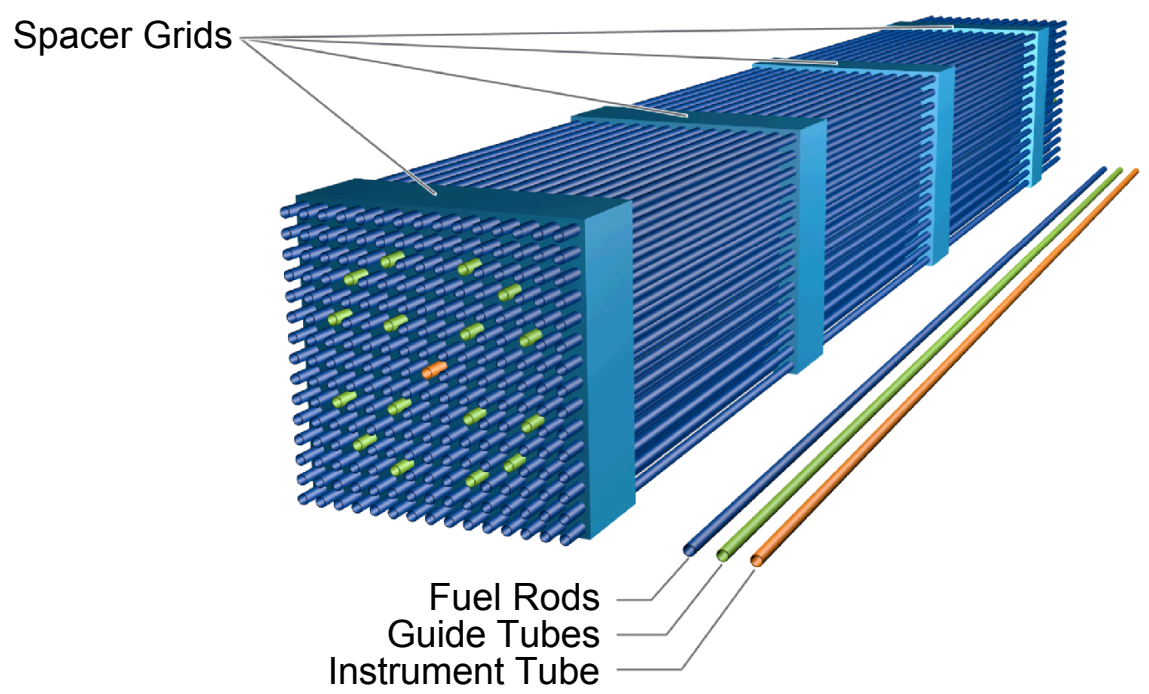

Figure 20. Representative fuel-element schematic showing how the ends of the fuel rods are accessed. (Graphic redrawn courtesy of the U.S. Department of Energy.) 
While it may be possible to bring in the signals through the side of the fuel rods, positioning and installing the transceiver antenna in the reactor would be more difficult. Integration of the micro-patch feedthrough into the side wall, as well as installation of the sensor, would also be more difficult. The outer fuel rods would prevent EM waves from penetrating into the center of the fuel element, thereby limiting in-core sensing to the outer fuel rods.

Adding the control rod assembly complicates the transmission path of the EM waves both to and from the sensors, as shown in Figure 21. The top nozzle blocks a considerable amount of EM signals from reaching the fuel caps. The flow orifices in the top nozzle provide windows for some EM signals to pass through and illuminate the fuel caps. Addition of the rod cluster control assembly to the fuel element shields flow orifices in the top nozzle from the transceiver located at the top of the reactor but also provides an opportunity to bring a transceiver closer to the fuel rods by routing it through the shaft of the control assembly, as shown in Figure 22.

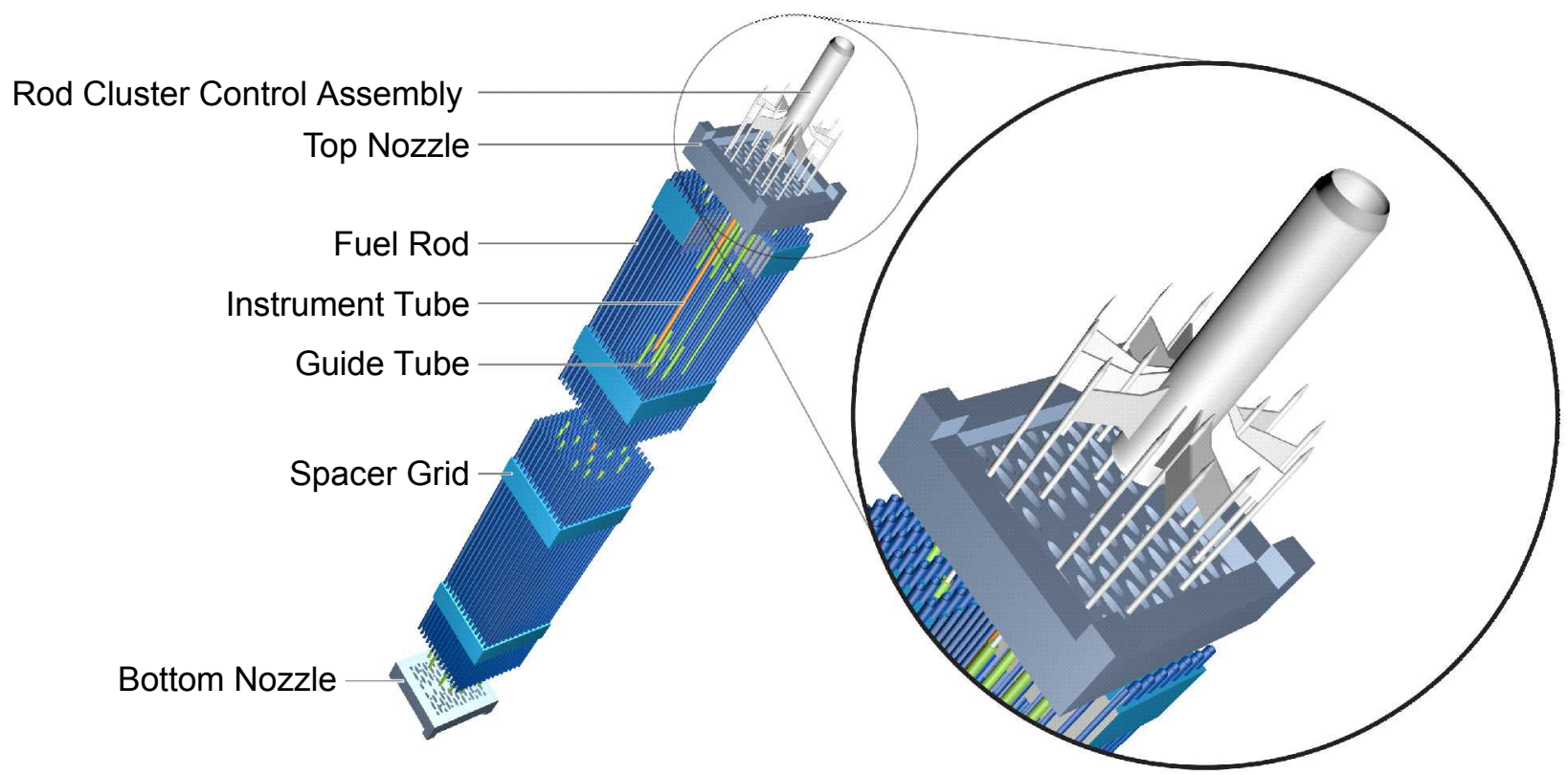

Figure 21. Schematic of the installation of the rod cluster assembly onto the fuel element, showing the EM shadowing of the fuel rod caps. The rod cluster control assembly shields flow orifices in the top nozzle from the transceiver located at the top of the reactor. However, the rod cluster does provide an opportunity to bring a transceiver closer to the fuel rods by routing it through the shaft of the control assembly. (Photo redrawn from Areva: https://www.sfen.org/sites/default/files/public/atoms/files/2012markus_birkhofer_areva_the_importance_of_the_fuel_design.pdf.) 


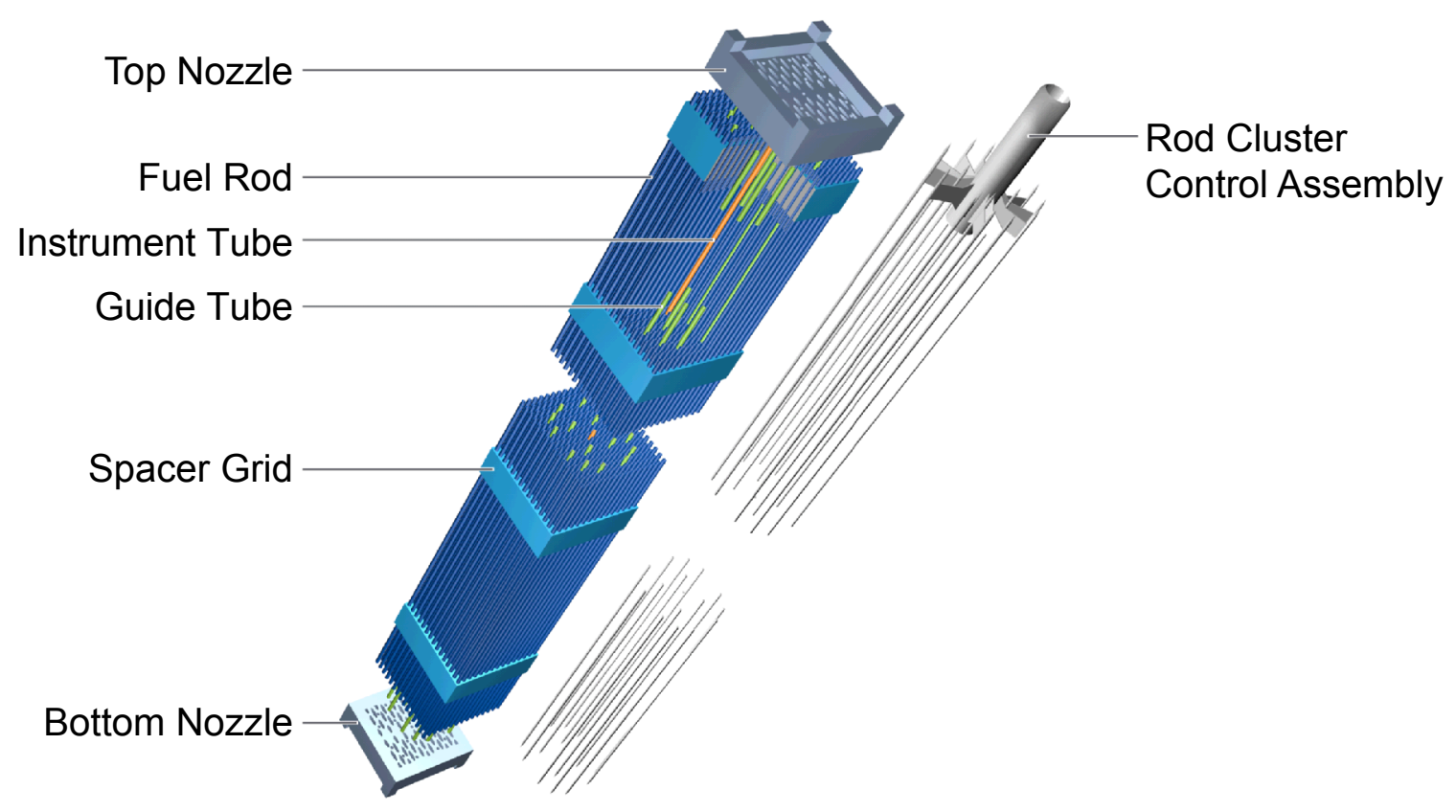

Figure 22. Schematic of the installation of the top nozzle onto the fuel element, showing EM shadowing of the fuel rod caps. Flow orifices in the top nozzle provide possible EM transmission paths. (Photo redrawn from Areva: https://www.sfen.org/sites/default/files/public/atoms/files/2012markus_birkhofer_areva_the_importance_of_the_fuel_design.pdf.)

The shaft of the rod cluster assembly is used to bring wiring to the instrument thimble. Figure 20 shows the location of the instrument tube in the fuel element that houses the instrument thimble. The shaft can either bring in the transceiver antenna via wires underneath the control rod fixturing or by using the hollow shaft as an EM waveguide to deliver and receive signals. Again, not all the fuel rod locations are useable as sensor locations. Laboratory mock-upsand modeling would determine the useful locations in the fuel element.

The inductive measurement techniques employ a substantially different strategy than the EM methods. The inductive measurements rely on using the instrument tube to house the active part of the sensor. Since the inductive standoff distances are limited to centimeters, the instrument tube location can be used to interrogate the fuel rods next to the instrument tube (see Figure 20). The inductive transmitter within the instrument thimble will excite the inductive sensor within a fuel rod (see Figure 17). The inductive receiver will then receive the signal and transmit it via wires through the shaft of the rod cluster control assembly.

\section{TRANSMITTING A SIGNAL FROM CONTAINMENT}

NPP containment buildings are safety-related structures with several important functions. In emergency situations, these structures contain escaping radioactive steam or gas, as well as protect the reactor from projectiles. Containment buildings in current working reactors usually consist of reinforced concrete with a metal lining that encloses the nuclear reactor [46-50]. The containment building is the fourth and final barrier to radioactive gas and contamination release; the first barrier is the ceramic, the second is the metallic cladding, and the third is the reactor vessel, including the coolant system [50]. All NPPs in the U.S. are designed to survive prescribed conditions specified as "design basis accidents" in the Final Safety Analysis Report, which is part of the operating license granted by the U.S. Government.

Containment buildings are usually gastight steel and concrete structures that encapsulate a reactor and 
isolate it from the outside atmosphere. The steel liner can either be free-standing or attached to a concrete projectile barrier. As indicated, the designs vary, depending on the reactor vendor and location. Figure 23 is a representative illustration of a working reactor containment building.

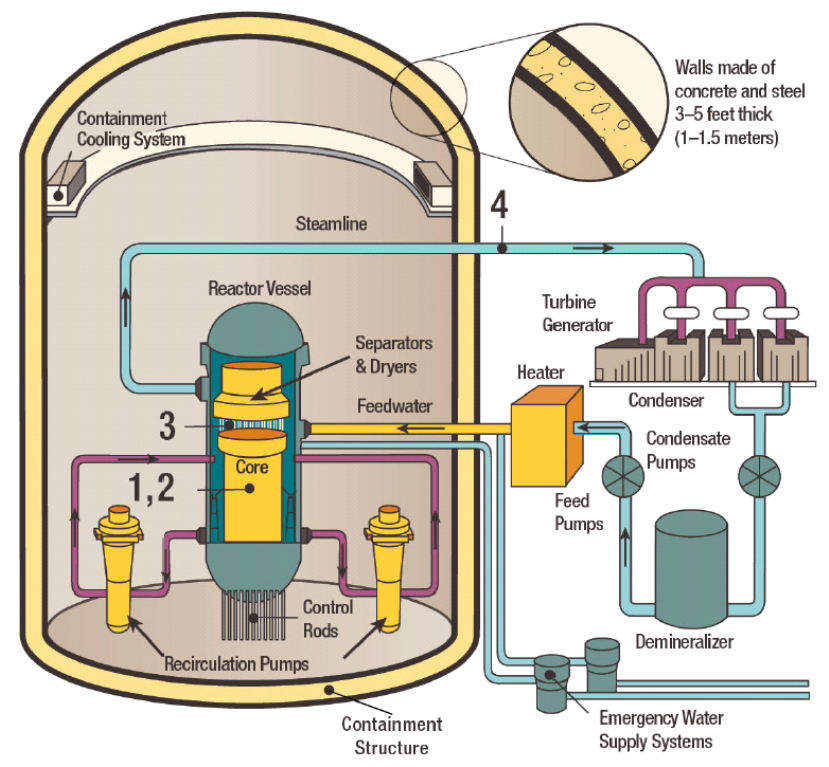

Figure 23. Schematic of a boiling-water reactor, showing the containment building. Figure courtesy of NRC [51].

As indicated in Figure 1, measurement signals from within the core will be conveyed into the containment building. The signals need to be either amplified or digitized, then transmitted outside the containment building to the balance of the plant communication system. The preferred method would be to digitize the signals, then transmit the digital data multiplexed from multiple sensors. Only monitoring data is considered for transmission in this report, as safety-critical and control signals have their own isolated secure transmission systems. Equipment to perform this transmission function would need to be installed and powered within the containment area.

All nuclear reactors, from current working reactors to proposed advanced reactor designs such as small modular reactors and microreactors, share similar functionality in terms of a traditional containment structure. The advanced reactors currently being designed have the advantage of being able to integrate the appropriate support infrastructure to process and transmit sensor signals to the balance of the plant communication system. This section focuses on transmitting measurement signals from the representative boiling-water reactor (shown in Figure 23) by retrofitting the necessary infrastructure.

\subsection{Structural Details of the Containment Building}

Working containment buildings are relatively large. For example, Plant Vogtle's containment structures are $140 \mathrm{ft}$ in diameter and 226- $\mathrm{ft}$ high [52]. The walls consist of near 4-ft-thick steel-reinforced concrete. The concrete walls contain large interlacing rebar, a steel cable system, and an interior steel plate. Fortunately, the containment structure should be large enough to support the necessary infrastructure if reasonably sized and if the space is efficiently used (see the video of the containment interior in [52]). Sensitive electronics can be shielded from radiation within the containment building. Unfortunately, the complexity and layered construction of the wall make it extremely difficult to transmit signals out of the containment building.

The difficulty in getting signals out of the containment building provides ample research opportunities. The gastight steel lining acts as a Faraday cage and makes standard RF transmission 
impractical. The reinforced concrete is highly attenuative to ultrasound. While communication techniques may be developed independently for each layer combining the wall layers drastically increases the difficulty of transmission. Note that reactor containment buildings can have significant construction variations. For example, some containment buildings may have significant air space between the steel lining and the concrete wall. Therefore, the transmission system would need to be customized for each reactor facility.

\subsection{Through-Wall Transmission Research}

A number of researchers are looking into opportunities afforded by the containment building. The Nuclear Energy Enabling Technologies program, initiated by the Department of Energy's Office of Nuclear Energy, is sponsoring several technologies through the Advanced Sensors and Instrumentation program. The most promising of the technologies take advantage of piping already installed in the reactor buildings.

Figure 23 is a representative schematic showing the types of piping contained within and exiting the containment building. Ultrasonic transmission systems based on transmitting ultrasonic waves via the pipe walls are being developed based on laser-generated [53] and standard piezoelectric transducers [54]. The ultrasonic waves can potentially travel long distances within an unconstrained pipe wall, but insultation, environmental covers, and airtight seals throughout the containment walls will significantly attenuate the ultrasound. Research is being conducted on getting measurement signals through the steel walls of a multipurpose spent fuel canister [55]. The instrumentation within the containment building could possibly be thermally powered by the heated pipes using a thermoelectric module similar to the one being developed in [55]. This may save valuable electrical cable space in the hermetically sealed electrical feedthroughs while also reducing installation costs.

\subsection{Using a Fluid Transmission Path}

Following a similar strategy of using the piping wall as a transmission path, the fluid within the piping can be used as an acoustic transmission path, potentially with direct access to the core (as suggested by Figure 23) and offering a convenient avenue out of the containment building. The piping functions as an acoustic waveguide, preventing acoustic energy from dispersing as it propagates to a receiver located outside the reactor. A thermoacoustic sensing system was developed to take advantage of the reactor environment and was demonstrated in a research reactor [56-63].

The thermoacoustic phenomenon provides for an all-in-one self-powering sensor with inherent wireless telemetry capability, an integrated transducer, and continuous measurement capability. A fuel pin can act as the acoustically resonant cavity, powered by the temperature gradient between the fuel pin and the cooling fluid. The thermoacoustic sensor acoustically radiates, filling the reactor vessel with sound. Some of the acoustic energy will make it into the pipes and be guided outside, where the acoustic signal can be detected and demodulated. The thermoacoustic sensor can be configured to measure neutron flux, temperature, or displacement. Multiple sensors can be frequency-multiplexed, and multiple signals can be received by a single receiver - such as an accelerometer or hydrophone. The receiver would be located outside the containment building in contact with the pipe or fluid. Since the acoustic signals travel within the fluid, hermetic seals and insulation minimally affect signal propagation. The received signal can then be transmitted to the balance of the plant communication system for broadcast to the control room or data cloud.

\subsection{Electrical Cable for Containment Transmission}

Though it is appealing to keep the transmission system wireless, multi-layer containment walls significantly impede through-wall transmission. As mentioned, researchers are developing technology to address this issue. Even if they are successful, this cutting-edge technology must compete with wired/cable transmission on both a practical and an economic basis. Major impediments to using cabled 
transmission are the costs of installing/routing the cables and their vulnerability to mishaps during normal plant operations and maintenance. For applications in the containment building, the impediments to using cables are significantly reduced. The cable runs would be relatively short - on the order of $500 \mathrm{ft}$. - and the potential for mishap is reduced by being within a limited space indoors.

Electrical cables have performed safely over the 40-year planned lifetime of the typical NPP, with few age-related cable failures recorded [64-66]. NPPs are now considering submitting applications to extend this lifetime period to up to 80 years [64]. It would be difficult for any other form of instrumentation to match the operating lifetimes and low need for maintenance that electrical cables are proven to offer. Because a cable can have a maximum bandwidth of $30 \mathrm{GHz}$ [67], a single cable would have ample communication bandwidth to transfer all the digital monitoring data. Reactor critical data from safety-critical systems, reactor control systems, etc., have their own, separate qualified transmission systems. Adding a single cable to the existing electrical feedthroughs should be feasible and would be a reasonable compromise. The advantages of using a single electrical cable to transmit signals out of the containment building may outweigh the potential benefits of wireless transmission. Ultimately, the decision will be based on the engineering tradeoffs necessary for the upgrades. For advanced reactors currently being designed, the possibilities are unlimited, as the transmission systems can be creatively built into the reactor structures.

\section{TRANSMITTING A SIGNAL FROM THE BALANCE OF THE PLANT}

NPPs are moving toward advanced technologies to automate monitoring, inspection, security, etc., for improved operating efficiency and effectiveness without compromising safety or reliability. In "Delivering the Nuclear Promise," the Nuclear Energy Institute, the Institute of Nuclear Power Operation, and the Electric Power Research Institute committed to making nuclear power generation one of the most competitive sources of energy in the U.S. market [68]. Although wired solutions present reliable channels for transmitting data, wired network deployment costs are extremely high. Industrial automation has becoming significantly more advanced with the emergence of $5 \mathrm{G}$ technology, as well as advanced lowpower IoT technologies.

Most currently available wireless technologies such as wireless local area networks (WLANs), Bluetooth, Zigbee, and RFID use the 2.4-GHz industrial, scientific, and medical (ISM) band. With the increased number of devices, the $2.4-\mathrm{GHz}$ ISM band is becoming overcrowded. Interference caused by different wireless technologies is increasing, resulting in high packet loss and low performance. Given the concerns associated with 2.4-GHz communication, expensive, Long-Term Evolution (LTE)-based solutions such as Narrowband-IoT, Category-1, and Category-M are evolving as low-energy, low-bit-rate solutions for IoT applications. In addition, proprietary technologies including long-range wide-area networks (LoRaWANs) and RFID are making their mark on IoT applications. These proprietary technologies use unlicensed spectrum bands (such as $900 \mathrm{MHz}, 2.4 \mathrm{GHz}$, etc.) with simple, cost-effective deployments. Additionally, these technologies will need either WLAN or cellular network backbones to upload/offload data.

With the diversity of wireless technologies available today, it is impossible to develop a one-size-fitsall solution to enable industrial automation. Different types of data are transmitted over wireless networks with different quality-of-service $(\mathrm{QoS})$, latency, and bandwidth requirements. In this regard, it is important to identify key aspects such as data rate, traffic type, distance, energy consumption, and network density in regard to designing a network that meets minimal industrial automation requirements. This will also ease data transmission and storage from the point of collection to remote locations for further processing [69]. Hence, to satisfy industrial automation and communication needs, co-existing heterogeneous networks (HetNets) should be built. For HetNet deployment, mutual interference between different wireless technologies must also be addressed. Unlike in other deployment scenarios for 
industrial environments, operators need centralized control over all the networks in order to reduce implementation costs, provide single-point control/monitoring of network devices, improve security, and enhance connectivity.

The choice of wireless technology will depend on technical features and capabilities such as coverage, capacity, latency, and QoS. The link budget, which defines the power loss in transmitting a signal through a medium, will be designed for the usage area and will determine the number of base stations, antennas, and access points (APs). The link budget will also determine the transmitter power and the separation between base stations and APs. Each frequency band-such as $2.4 \mathrm{GHz}$ for WLAN or $700 \mathrm{MHz}$ for LTE — have different link budgets, making network designs specific to each type of wireless technology. If more than one wireless technology is considered, mutual interference must also be considered when building a co-existing HetNet to support a wide range of applications and user requirements. In this regard, distributed antenna technologies such as distributed antenna system (DAS) and WLAN technologies are widely used in industrial communication. DAS is widely used to provide cellular connectivity in both indoor and outdoor environments. In industrial environments, either WLAN or cellular networks are integrated with a DAS unit. This integration can be used to upload/offload data to a remote location or cloud for data analysis, monitoring, diagnosis, and prognosis.

\subsection{Network Architecture}

Most network topologies are predominantly driven by DAS or WLAN systems, since they can enable (1) high bandwidths and high data rates with low latency, (2) prioritized data transmission based on the required QoS, and (3) consolidation of multiband wireless technologies under one network architecture. This report focuses on a representative DAS wireless network. The next section summarizes the features and components of a DAS wireless network architecture, as shown in Figure [70].

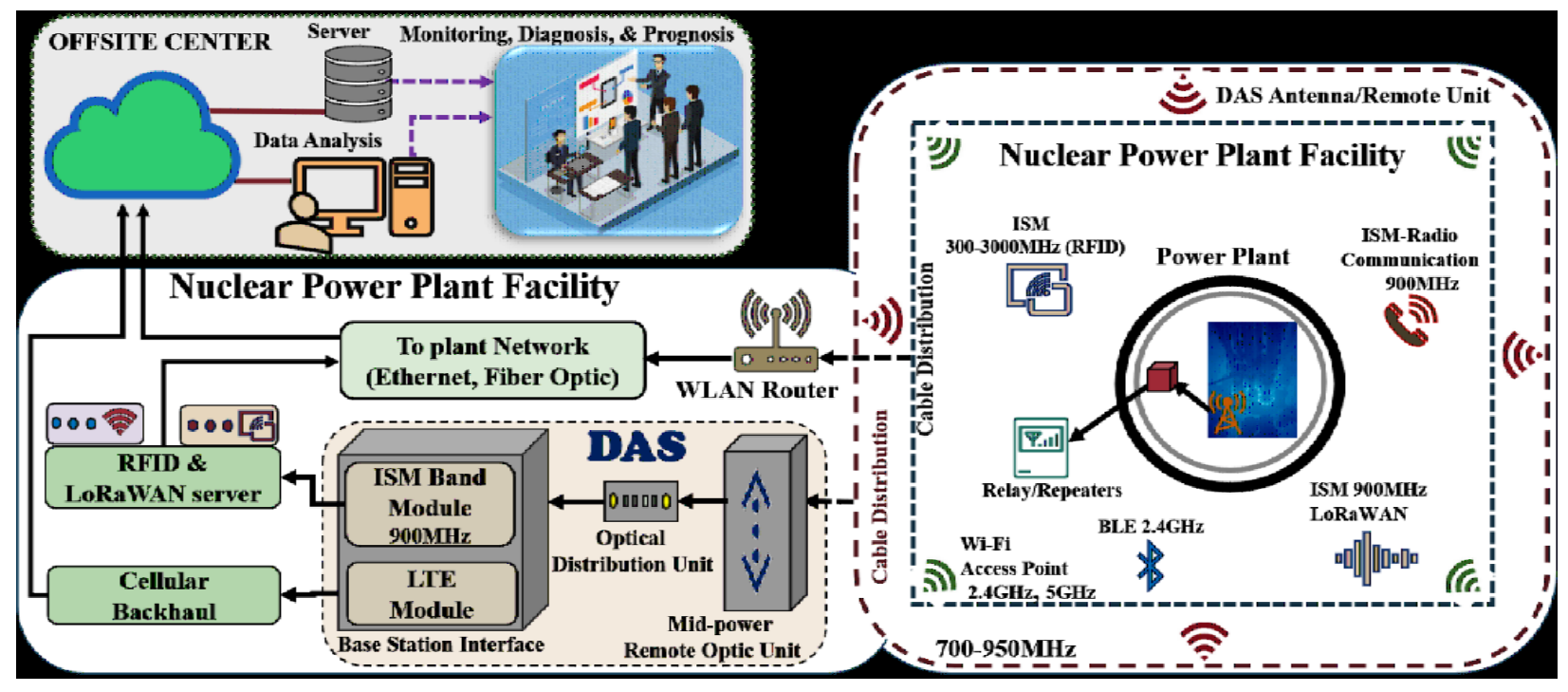

Figure 24. Envisioned DAS wireless network architecture for NPP automation.

\subsection{Distributed Antenna System}

In this report, a digital DAS system is considered, consisting of a main unit (MU), expansion unit (EU), remote unit (RU), and antenna elements (AE). These components are interconnected using optical fiber cables of appropriate lengths at different height levels. Fiber-optic cables connect the MU to the EUs. For a DAS system, a maximum of four EUs can be connected to the MU. EUs and RUs are connected using Category-5 or coaxial cables. A thin coaxial cable connects the RU to the AE. The length of the Category- 5 or coaxial cables depends on the number and placement of the AE. It is important to 
note that the RU itself can have embedded AE.

In Figure 24, the sensor nodes using cellular technology (LTE) send measurement data to the closest antenna. The received RF signal is distributed to independent electrical amplifiers with the appropriate frequency band. The amplified independent signals are then multiplexed and fed into the Mid-Power Remote Optic Unit (MPROU), which converts the multiplexed RF signal into an optical one. The optical signal is then fed into the Optical Distribution Unit (ODU), where optical signals from several MPROUs can merge. Note that the signals from the MPROU to the ODU are optical because MPROUs can be located in remote locations at the facility. The optical cables help carry the signal from the remote location to the front end of the DAS without attenuation. At the ODU, the optical signals are converted back into RF signals and received at the base station interface (BSI). At the BSI, the RF signals are filtered at the appropriate frequency bands, then the signal is sent to the relevant carrier's cellular backhaul. Note that each cellular carrier uses different frequency bands, therefore filtering is needed to separate the signals. From the cellular backhaul, the signal goes through the packet core network of an individual carrier and over to the cloud for storage, monitoring, and diagnosis.

It is important to note that, for clarity, the data flow in Figure 24 is shown to be unidirectional from the reactor core. In actuality, two-way communication to the instrumentation is also a possibility. Twoway communication allows for remote control of the monitoring instrumentation in order to enable the ability to remotely change settings and conduct trouble-shooting remotely.

A DAS testbed for information uploading was deployed at INL's Human System Simulation Laboratory (which is at TRL-4), as shown in Figure 25. The testbed was developed using DAS components from solids [71]. The testbed consists of an MU, EU, and a single RU with an AE. The transmission power from the BSI ranges from $-20 \mathrm{dBm}$ to $+10 \mathrm{dBm}$, with variable attenuation of up to 30 $\mathrm{dB}$. The 5 -W MPROU delivers $+37 \mathrm{dBm}$ of output power per band at the antenna port for all commercial bands. This 5-W MPROU can simultaneously support seven bands with a frequency range of $136 \mathrm{MHz}$ to $2.6 \mathrm{GHz}$, with an add-on RU to support very-high-frequency and ultra-high-frequency bands.
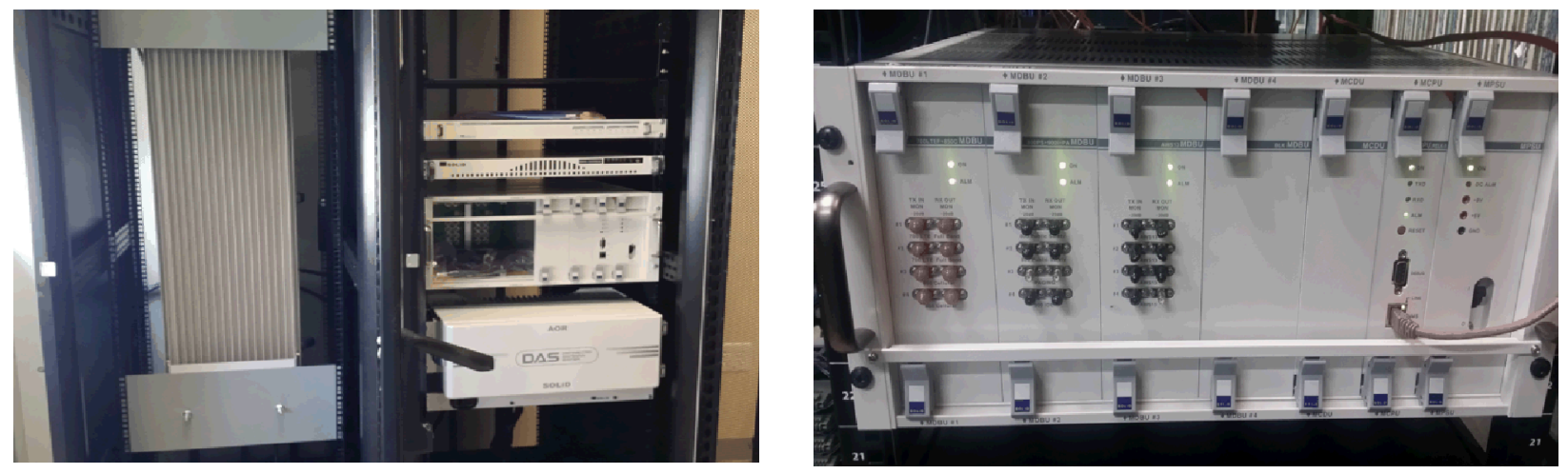

Figure 15. DAS testbed installation at INL's Human System Simulation Lab. Left: MPROU, Right: BU

\subsection{Wireless Local Area Networks}

Sensing systems that use WLAN technologies to broadcast data can also be integrated into the communication infrastructure. As indicated in Figure 24, WiFi access points are distributed throughout the plant. The measurement data are broadcasted to a WLAN router connected to an internal plant network. The in-plant network delivers the measurement data to the data cloud.

WLAN [73] features low coverage and limited-capacity access points. WLAN operates at unlicensed spectrums of $2.4 \mathrm{GHz}, 5 \mathrm{GHz}$, and $6 \mathrm{GHz}$ capable of providing high-data-rate internet connections.

WLAN distributes the network capacity by allotting wireless APs with limited frequencies. This is similar in principle to cellular networks, fostering the ability to fulfill coverage and capacity requirements. Since 
WLANs operate at unlicensed bands, interference will most likely be caused by neighboring APs. It is necessary to have transmission power control to properly distribute the APs. The sixth-generation WiFi standard 802.11ax [73, 74], also known as "WiFi-6," is the latest step in WLAN advancement. WiFi-6 is aimed at high flexibility and scalability. This standard enables expansions to include new and existing networks for powering next-generation applications. WiFi-6 enhances network throughput due to advanced orthogonal frequency division multiple access (OFDMA) and multi-user multi-input multioutput (MU-MIMO) with beamforming capability. WiFi-6 uses the higher modulation enabled by 1024-QAM. With MU-MIMO capability, WiFi-6 can simultaneously transmit eight different spatially directed streams. In addition, WiFi-6 also supports low-power and low-bandwidth applications using a 2$\mathrm{MHz}$ channel bandwidth. WiFi-6 can use flexible low-power device scheduling with Target Wakeup Time. Moreover, in the 2.4-GHz band, the 20-MHz allocated bandwidths can be divided into nine 2-MHz channels. Any 2-MHz channel can be left unused to avoid conflicts with other existing IoT technologies such as Bluetooth Low Energy, ZigBee, etc. WiFi-6 also has built-in security strategies.

With NPP deployment, the WiFi APs are distributed across different sections of the NPP with proper transmit power control. The sensor node using the WiFi technology will initially be in idle mode to save power. When data is available to transmit, it will change from idle to active. When active, the sensor node receives the beacon signal sent by each AP at 1-ms intervals. The sensor node determines the SNR level of the received beacon signal and connects to an AP that has a maximum SNR. The connection request from the sensor node will be approved by the relevant AP. Once the connection is established, the sensor node starts transmitting the measurement data. The received data at the AP will be sent via ethernet cable to the router in the form of a data packet. The router routes the received data packet to the connected modulator-demodulator (MODEM) device. The MODEM uses a digital-to-analog converter to convert the received packets into a baseband analog signal, then modulates that signal. The modulated signal will be sent via coaxial or optical cable to the internet service provider and then to the cloud.

\subsection{Industrial, Scientific, and Medical Band Technologies}

Several wireless technologies such as LoRaWAN [75], RFID [76], etc., operate in the unlicensed ISM band (900 MHz in North America) for low-power, low-bit-rate, and long-range applications. Each technology has its own physical layer design, Media Access Control (MAC) layer protocols, and security algorithms. Two common applications that utilize this ISM band are (1) device tracking via RFID and (2) device monitoring and control using LoRaWAN. As stated earlier, these technologies can be integrated along with cellular technology using a DAS structure. Besides interconnecting other networks such as Bluetooth Low Energy and Zigbee, sensor modules such as video surveillance and mmWave radar can be connected to either cellular or WLAN networks, without additional expensive cable installations.

ISM-band technologies such as RFID and LoRaWAN front-end are connected to the DAS structure. Sensors using LoRaWAN or RFID will send the signal through the DAS antenna. Like a cellular signal, the ISM band signal will go through MPROU as an RF signal, then to ODU as an optical signal. At BSI, the filters for the ISM band extract RFID and LoRaWAN signals, and these are sent to their respective servers. At the server, the received RF signal is converted into data packets. The back-ends of the RFID and LoRaWAN servers are connected to the plant's ethernet, over which the converted data packets are transmitted to the cloud through the router and MODEM.

\subsection{Relay/Repeater Configuration}

The repeater is a device that receives the signal at one end, performs the necessary conversion of the data format, and transmits at the other end. In this configuration, the repeater receives RFID data at the front-end and forwards them to the cloud through DAS/WiFi at the back-end. The relay node employs a signal conversion mechanism in which the received signal is down-converted to a baseband signal. The baseband signal is converted into digital packets, then internet protocol, MAC, and payload information are extracted. Note that the MAC information is unique to each device and determines the device's 
physical address. Using the transmission control protocol (TCP)/IP protocol, the relay node sends feedback to the RFID node every time it receives a payload. This feature is necessary to ensure that the data received from the RFID node is free of errors. After verifying the payload, the payload is appended with an IP address and the MAC information of the relay node. This constitutes a "packet." The packet is converted into an analog baseband signal using a digital-to-analog converter. The baseband signal is then up-converted using a modulation scheme specific to wireless communication protocols. For example, WiFi and Cellular LTE use quadrature amplitude modulation with an OFDMA mechanism, whereas LoRaWAN uses spread-spectrum modulation. The modulated signal will be transmitted to the cloud either by DAS antenna (if the relay uses cellular/LoRaWAN) or WiFi AP.

The main advantages of the proposed network architecture in Figure 24 are (1) efficient deployment and modification, (2) scalability, (3) customizable network architecture and security options, and (4) network resiliency. Communication network resiliency will enable multi-path routing if an AP or DAS antenna fails or becomes jammed. A nearby AP or DAS antenna can receive the measurement data and dynamically re-route the signals to the cloud.

Table 1 compares different wireless technologies that enable industrial automation in NPPs. Both LTE and WiFi-6 support higher data rates using an OFDMA modulation scheme with MU-MIMO, whereas RFID, LoRaWAN, and BLE-5 support lower data rates and use either LTE or WiFi-6 to send the data to the cloud. WiFi-6 and LTE have shorter ranges, while the other technologies can transmit data over longer ranges. All the technologies provide different levels of security for transmitting data with minimal risk. Since LTE and WiFi-6 provide IP address capability, complex and sophisticated security protocols can be enabled for those standards. Applications such as RFID that have low bitrates and infrequent communication bursts can extend battery life. Note that wireless technologies are not limited to those listed in Table 1; other technologies such as Zigbee, SigFox, etc., can also be considered.

Table 1. Comparison of different wireless technologies.

\begin{tabular}{|c|c|c|c|c|c|}
\hline & LTE & WiFi-6 & BLE-5 & LoRaWAN & RFID \\
\hline $\begin{array}{c}\text { Data } \\
\text { Throughput }\end{array}$ & $\begin{array}{l}\text { Up to } 10 \\
\text { Gbps }\end{array}$ & $\begin{array}{l}\text { Up to } 4.8 \\
\text { Gbps }\end{array}$ & Up to $2 \mathrm{Mbps}$ & Up to $50 \mathrm{Kbps}$ & $\begin{array}{c}\text { Up to } \\
128 \mathrm{Kbps}\end{array}$ \\
\hline Range & $500 \mathrm{~m}$ & $100 \mathrm{~m}$ & Up to $750 \mathrm{~m}$ & $10 \mathrm{~km}$ & Up to $300 \mathrm{~m}$ \\
\hline $\begin{array}{c}\text { Power } \\
\text { Consumption }\end{array}$ & A few hours & $\begin{array}{c}\text { Up to } 1 \text { year } \\
\text { on AA } \\
\text { batteries }\end{array}$ & $\begin{array}{l}\text { Up to years on } \\
\text { a coin cell } \\
\text { battery }\end{array}$ & $\begin{array}{l}\text { Up to years on } \\
\text { a coin cell } \\
\text { battery }\end{array}$ & Over 6 years \\
\hline $\begin{array}{l}\text { Frequency } \\
\text { Band }\end{array}$ & $\begin{array}{c}600 \mathrm{MHz}-6 \\
\mathrm{GHz} \text { and } 24 \\
-86 \mathrm{GHz}\end{array}$ & 2.4 and $5 \mathrm{GHz}$ & $2.4 \mathrm{GHz}$ & $\begin{array}{c}902-928 \mathrm{MHz} \\
\text { (U.S.) }\end{array}$ & $\begin{array}{c}120 \mathrm{kHz}-10 \\
\mathrm{GHz}\end{array}$ \\
\hline Modulation & OFDM & OFDM & GFSK & $\begin{array}{c}\text { Spread } \\
\text { Spectrum }\end{array}$ & $\begin{array}{c}\text { ASK, PSK, or } \\
\text { FSK }\end{array}$ \\
\hline IP at the Device & Yes & Yes & No & No & No \\
\hline Licensed & Both & $\begin{array}{l}\text { Unlicense } \\
\text { d }\end{array}$ & $\begin{array}{l}\text { Unlicense } \\
\quad \mathrm{d}\end{array}$ & Unlicensed & $\begin{array}{l}\text { Unlicense } \\
\quad \mathrm{d}\end{array}$ \\
\hline Security & $\begin{array}{l}\text { NAS/A } \\
\text { S }\end{array}$ & $\begin{array}{l}\text { WEP, WPA } \\
\text { etc. }\end{array}$ & 128-bit AES & 128-bit AES & Available \\
\hline
\end{tabular}

FSK: frequency shift keying, PSK: phase shift keying, ASK: amplitude shift keying, GFSK: Gaussian 
frequency shift keying, OFDM: orthogonal frequency division multiplex, WEP: wired equivalent privacy, WPA: WiFi protected access, AES: Advanced Encryption Standard.

\section{WIRELESS PLAN GOING FORWARD}

This plan presents a wireless sensing and communication system for use within a reactor core. Ceramic-based high-temperature sensors that are wireless and passively powered will be integrated into a communication network to enable delivery of in-core data to a cloud database and a data processing center. The system will provide real-time sensing for in-core nuclear applications subject to hightemperature, high-pressure, high-radiation fluxes and oxidation challenges. This will require the use of sensors that are robust with respect to high-radiation and high-temperature environments. The goal is to collect real-time information from sensors located throughout the reactor, so the reactor core and fuel elements within can be monitored cost-effectively using precise, localized measurements. The hightemperature- and radiation-resistant sensors will be suitable for a variety of reactor types (e.g., LWRs, gas reactors, etc.).

An advantage of PDC sensors is their multi-sensing capability for simultaneously measuring temperature, pressure, strain, and various other parameters. The sensors will be frequency-multiplexed, with multiple micro-patch antennas designed to resonate in different frequency bands. An advanced communications system will be developed in which multiple sensors can be interrogated directly. Instrumentation to demodulate, process, and digitize the data will be located in a more hospitable environment within the containment building.

To meet the plan goals, development of the wireless sensing and communication system will be phased according to the following stages. The individual technologies, RFID sensors and infrastructure, and the balance of the plant communication network are currently at TRL-4.

- Each technology will require modification to integrate with the other technologies.

- RF sensors will need to be integrated into the RFID infrastructure.

- The RFID infrastructure will be integrated with the balance of the plant network.

- TRL-4 testing will be conducted for the fully integrated wireless sensing and communication system.

At every stage, the sensing system will be tested to ensure measurement quality and communication system efficiency. From the initial sensor stage consisting of lab RFID instrumentation to TRL-4 testing of the fully integrated system, all subsystems and integrated systems will be quality tested. The RFID infrastructure will be designed to multiplex multiple sensors. A multiplexing system with at least six multiplexed sensors will be designed and benchmarked against industry-standard thermocouples. Temperature testing will take place in furnaces at the various facilities where the integration is taking place. It is anticipated that the fully integrated system will be TRL-4-tested using an autoclave at INL's HTTL.

The sensor has salient design parameters that will feed forward into the RFID infrastructure. The resonant frequency for a ceramic sensor in a reactor's working environment falls in the range of 1-10 $\mathrm{GHz}$. The specific centerband frequency and frequency range for each sensor will be decided in Stage 1 . The transmitter power and the power received at the sensor will also be decided in Stage 1. The estimated minimum power needing to be received by the sensor in order to be fully functional is $0.363 \mathrm{~mW}$ in free space at $5 \mathrm{GHz}$. The in-situ temperature and pressure conditions inside the reactor core will be collected in real-time (sampling rate of at least $1 \mathrm{~Hz}$ ). 


\subsection{RFID Sensing Infrastructure}

The RFID sensing infrastructure consists mostly of the RF interrogator, which operates as an intermediary layer for communication between the passive RF sensor node inside the fuel rod and the balance of the plant network. The interrogator can be installed in the containment building outside the pressure vessel. The interrogator has its own power source, a reliable connection to the balance of the plant network via LAN or WiFi over a TCP/IP network, and a coaxial wired connection with a transceiver antenna inside the reactor pressure vessel. A schematic of the RF interrogator is shown in Figure 26.

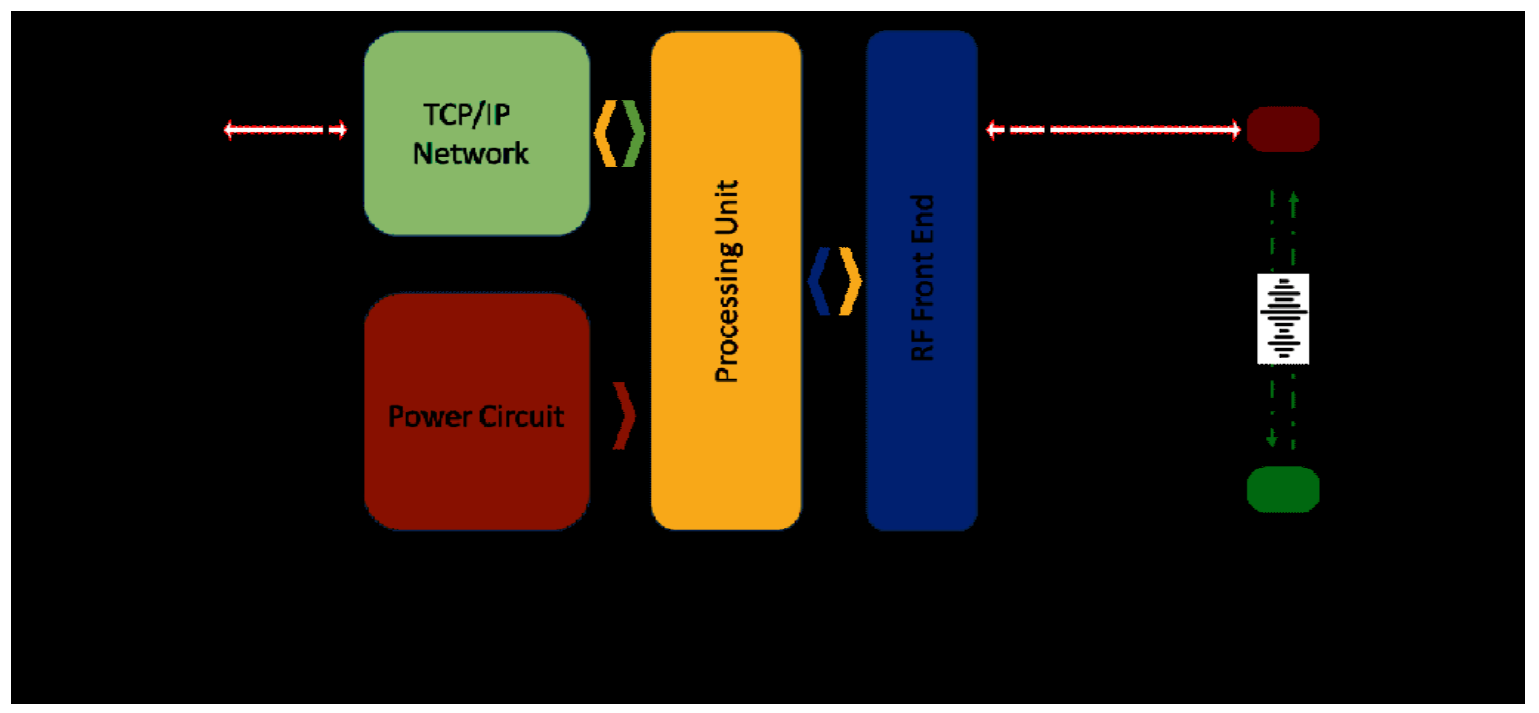

Figure 26. Schematic of an RF interrogator for wireless communication with the passive RF sensor node and the relaying of information to the balance of the plant network.

The RF interrogator can transmit over a range of frequencies (1-5 GHz) to wirelessly power the passive RFID sensor via a transceiver antenna that is $3 \mathrm{~cm} \times 3 \mathrm{~cm}$. The passive RFID sensor backscatters the RF signal with modulated sensor information. The backscattered signal is acquired by the RF interrogator. The interrogator's data acquisition system demodulates the passive RFID sensor signal, digitizes it, and processes it to extract precise sensing information. The processed digital data is reformatted and stored in local memory, ready for transfer to the balance of the plant network over a TCP/IP network. Data from the RF interrogator can be transferred in real-time to the balance of the plant network at a sample rate of $1 \mathrm{~s}$.

Development of the RF interrogator system will feature the following milestones:

1. $\quad$ PDC sensor integration with the RF node: Passive RF nodes can have single or multiple sensing elements (up to six). For this work, a PDC-based sensor that changes its resistance according to the surrounding temperature will be connected to an RF antenna, shifting the antenna's resonance frequency due to impedance loading. As a result, the reflected or backscattered signal will be modulated accordingly. The PDC measurements will be benchmarked against those of traditional wired sensors.

2. Designing the wideband transceiver antenna and RF front-end: A wideband transceiver antenna for wireless communication will be designed to reliably operate inside the reactor pressure vessel while immersed in a liquid medium. The RF front-end will transmit continuous RF power at a frequency range of $1-5 \mathrm{GHz}$. An appropriate amount of RF power can be radiated using the transceiver antenna to actuate the passive RF sensor node at the estimated minimum required power level of $0.363 \mathrm{~mW}$. Additionally, it will be able to receive the reflected signal and demodulate the sensor information. 
3. Information digitization and TCP/IP transmission: The demodulated signal from the RF frontend will be digitized and processed using the RF interrogator's onboard resources. The processed signal will be stored in local memory before transmission over a TCP/IP network. A network chip will be integrated with the processing unit for realizing standard TCP/IP communication external to the RF interrogator.

4. Testing the RF interrogator at Michigan State University (MSU): For testing the communication range and sensing capacity of the RF interrogator, the passive RF sensor node with an integrated temperature sensor will be placed at distances of $0.1-1 \mathrm{~m}$ from the transceiver antenna encompassed in the liquid medium. A real-time reading of the temperature sensor will be shown on a computer connected to the RF interrogator. The PDC measurements will be benchmarked against those of traditional wired sensors. Moreover, the TCP/IP communication method will be demonstrated by plotting the temperature data on a simple web API, using a different computer connected to the same network or internet server.

\subsection{Balance of the Plant Network}

This plan presents a balance of the plant network system for use throughout the plant's infrastructure. It also includes getting the data from the sensors installed within the reactor core and containment building. The reactor core data is stored within the RFID infrastructure inside the containment building. RFID sensor data can be transmitted to a relay/repeater device kept close by outside the containment building. The communication protocol can be a serial protocol such as TCP/IP. The serial protocol allows for a single cable to transmit the data, along with existing containment electrical cable trays and wall feedthroughs. If the containment building has an accessible network, the sensor data might be directly transferrable to the plant's network.

As previously discussed, the technology to wirelessly transmit data through the containment walls is currently in the initial stages of research. Thus, the connectivity from the RFID infrastructure inside the containment building to the repeater will initially consist of a single coaxial cable connection. The repeater converts the received digital data into a wireless-specific protocol. Then the repeater node transmits the converted data to the closest working DAS antenna, and the signal from the antenna goes through the plant's DAS infrastructure. Note that the repeater can also be connected to a WiFi AP. The WiFi AP can deliver the data to the cloud via the plant LAN network. At the plant's DAS back-end, the signal is filtered using the appropriate frequency band. The filtered RF signal will be uploaded to the cloud over the cellular backhaul or through the cable ISP, as shown in Figure 27.

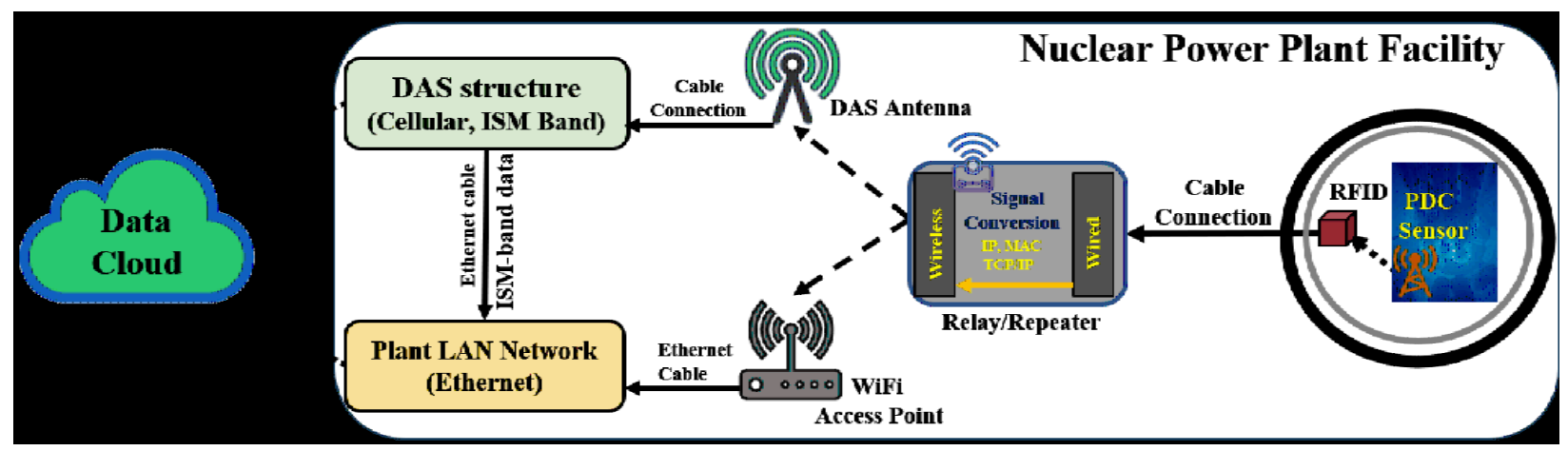

Figure 27. Data flow from the reactor core to the cloud using the balance of the plant network.

To achieve the above-mentioned functionality, a software-defined radio (SDR) will be used to develop a relay/repeater node. SDRs support both wired and wireless transmissions. The main advantage of using an SDR is that all the network configurations are programmable and support a frequency range of $\mathrm{MHz}$ to GHz. This capability enables connection to a relay/repeater node from a WiFi AP or DAS 
antenna. The choice will be based on signal quality and network congestion. This HetNet technology capability with relays/repeaters is advantageous in compensating for equipment failures and adverse data transmission conditions. HetNet also enables meeting QoS and bandwidth requirements. In addition, the SDR can be used to develop customized security protocols at both the wired and wireless connections.

To meet the plan goals, development of the balance of the plant network will be phased according to the following stages.

1. Developing a TCP/IP signal emulator: An RFID signal with a TCP/IP protocol will be emulated using a signal-generating device. Signal-generation characteristics in terms of bitrate and bandwidth will match the output protocols of the RFID infrastructure. This enables parallel development of the RFID infrastructure and balance of the plant network.

2. Developing HetNet wireless configuration: At the back-end of the SDR a HetNet wireless configuration supporting WiFi and DAS architecture will be developed. This configuration will be tested by connecting to INL's DAS testbed and WiFi.

3. Modify the existing communication system to integrate the RFID infrastructure: Using the SDR device at the front-end, the necessary configurations will be developed for the physical, MAC, and network layers. The developed configurations will decode signals and extract network-related information such as IP and MAC. The modified configurations will be tested using the TCP/IP signal emulator. Any necessary modifications to the SDR-based relay/repeater configuration will be addressed. Data transmission will be tested at every stage using signal analyzers.

4. Integrate RFID infrastructure with the developed SDR-based relay/repeater module: The developed SDR-based relay/repeater device will be integrated with the RFID infrastructure developed at MSU. The fully integrated wireless sensing and communication system will be tested at INL's communication lab at near room temperature. As part of the system testing, the end-to-end performance, network delay, and received data quality will be analyzed.

5. Testing at HTTL: The fully integrated wireless sensing and communication system will be installed in INL's HTTL, and the RFID sensor will be tested within an autoclave. Placement of the relay/repeater can follow multiple configurations. Currently, the most effective NPP deployment configuration is as follows: the relay/repeater is placed in the HTTL, and the relay is connected to INL's wireless link. Measurement data will be transmitted to the cloud housed in another building several blocks away. As part of the system testing, end-to-end performance, network delay, and received data quality will be analyzed. The RFID sensors will also be benchmarked against traditional wired sensors.

\section{WIRELESS RESEARCH AND DEVELOPMENT ROADMAP}

In this section, a technical roadmap is discussed for deploying battery-less (passive) sensing and communication technology for a Reactor Pressure Vessel (RPV) that addresses future challenges. The passive system is ideal for nuclear applications, as it requires no maintenance during its lifetime and can operate from inside the RPV without a wired connection. Figure 28 shows an RPV with integrated passive multi-sensing nodes in various locations. The RPV is the largest, most important structure in an NPP, as it houses the coolant and nuclear core. It is typically made of high-quality carbon steel and stainless steel. The wall thickness of an RPV varies from 150 to $400 \mathrm{~mm}$, and the operating temperature and pressure range as high as $500^{\circ} \mathrm{C}$ and 200 bar, respectively, depending on coolant type. 


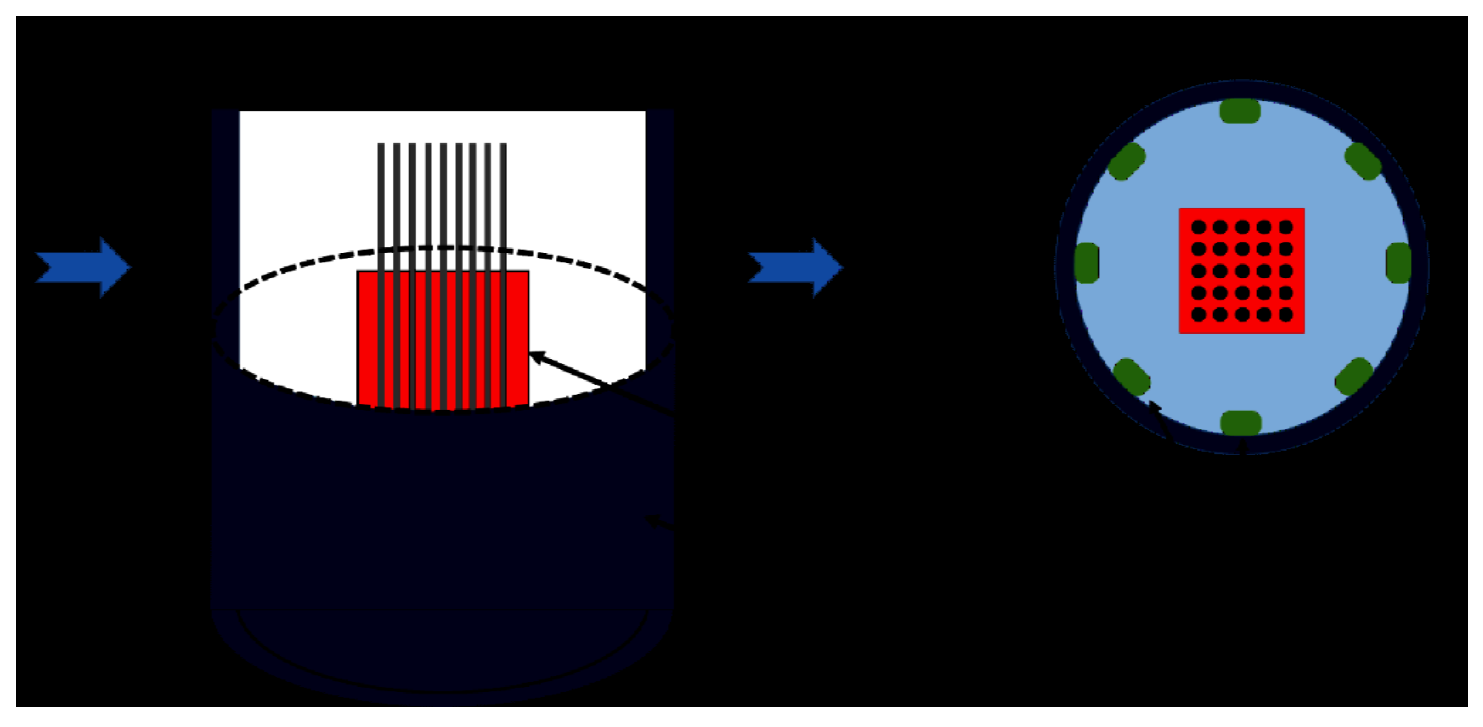

Figure. 28. Simplified model of a nuclear reactor pressure vessel and the eight battery-less sensing nodes integrated inside featuring radiation-hardened electronics and high-temperature sensors.

\subsection{Sensor Integration}

Each passive sensing node can house multiple resistive sensors (up to 6 in this version). The sensors relate to temperature, pressure, strain, etc. In this report, a PDC-based high-temperature sensor was selected in order to explain the sensor integration [20]. PDC temperature sensors can survive in up to $1500^{\circ} \mathrm{C}$ and offer excellent linearity. Additionally, the effects of radiation on ceramics are negligible. Changes in resistance due to temperature can be measured using the passive sensing node, which generates a corresponding unique sinusoid signature. The frequency of the sinusoidal signal varies according to temperature, and this signal can be wirelessly communicated to an interrogator. The mechanism for wireless communication from inside an RPV is discussed in the next section. A number of nodes can be installed inside the RPV, containing numerous sensors to measure various reactor parameters at multiple locations. The electronics required to process incoming commands and sensor signals must be radiation-hardened. This particular sensing configuration and corresponding capabilities would also enable testing and monitoring of materials and components (such as electronic circuits) while during irradiation.

\subsection{Wireless Communication from Inside the RPV}

An interrogator is required to communicate with the passive sensing nodes installed inside the RPV. No RF signal can penetrate a $140-400-\mathrm{mm}$-thick steel wall. Therefore, a novel acoustic interrogator is required to send and receive information from the sensors. The acoustic waves propagate via the steel wall without any problem and can transfer the power required to operate the battery-less sensing node. The acoustic bandwidth limits faster communication but is sufficient for real-time monitoring of conditions inside the RPV at $1 \mathrm{~Hz}$. The acoustic interrogator also has an active RF communication circuit to relay the information to a supervisory control and data acquisition system. Figure 29 shows the configuration of various interrogators and the sensing node placement. Both devices communicate through the steel wall. 


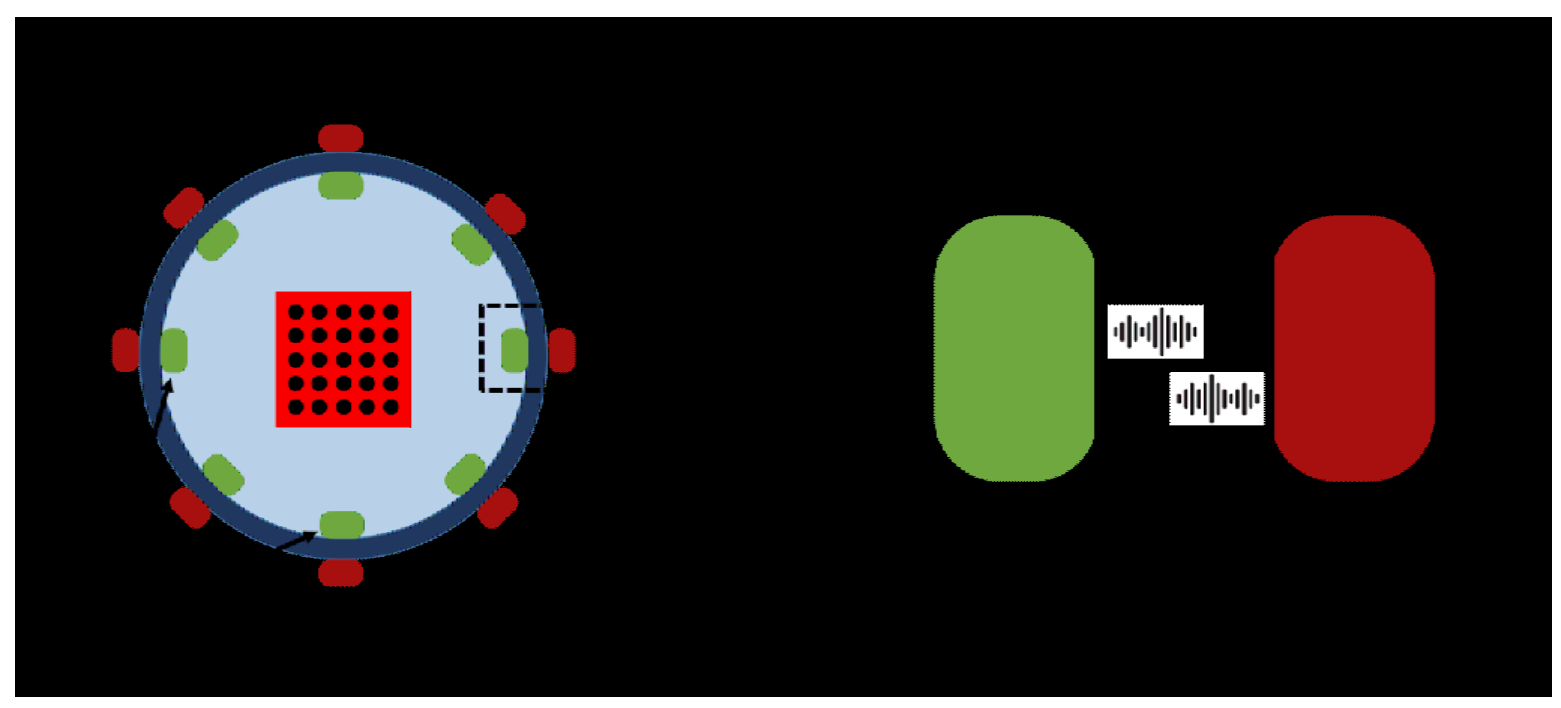

Figure 29. Acoustic interrogator for wirelessly communicating inside the RPV.

The acoustic communication system will resemble the two battery-less monitoring topologies explained above. The key difference lies in replacing the RF antenna with an acoustic transducer array capable of receiving enough power to actuate the monitoring circuit and transmit back the information. Figure 30 shows an example architecture for a battery-less sensing node based on the acoustic communication system proposed by MSU. The components shown in Figure 30 were previously realized for different applications [11] and can be further refined for nuclear applications. The acoustic power harvester, backscatterer, and processing unit circuits can be miniaturized into a small footprint with radiation-tolerant packaging. Additionally, the acoustic waves can be used for communication among the sensor nodes by integrating multi-sensors within the RPV. The acoustic signal can be transmitted through the coolant at communication distances of up to several meters. This is similar to underwater sonar. Therefore, the sensors can be placed anywhere inside the RPV, and not simply near wall locations, as long as the line of sight can be maintained.

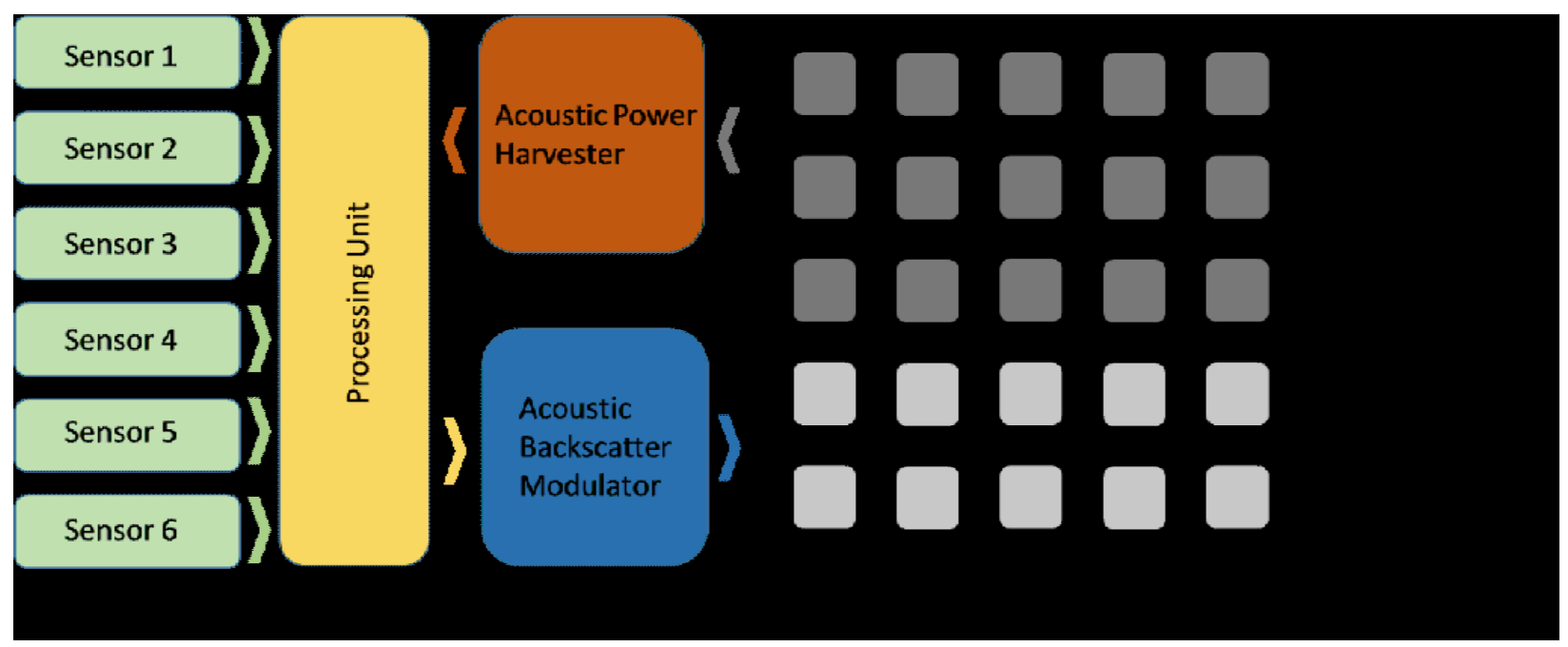

Figure 30. Architecture of the battery-less sensing node with a wireless acoustic communication system. 


\section{REFERENCES}

1. Kumar, D., Mondal, S., Karuppuswami, S., Deng, Y., and Chahal, P., "Harmonic RFID communication using conventional UHF system," IEEE Journal of Radio Frequency Identification, vol. 3, no. 4, pp. 227-235, December 2019.

2. Mondal, S., Kumar, D., Mohd. Ifwat, G., Chahal, P., Udpa, L., and Deng, Y., "Monitoring and localization of buried plastic natural gas pipes using passive RF tags,"” In 2018 AIP Conference Proceedings (Vol. 1949, No. 1, p. 020020). AIP Publishing.

3. Cheng, H., Shao, G., Ebadi, S., Ren, X., Harris, K., Liu, J., Xu, C., An, L. and Gong, X. (2014). Evanescent-Mode-Resonator-Based and Antenna-Integrated Wireless Passive Pressure Sensors for Harsh-Environment Applications. Sensors and Actuators: A. Physical, 220, 22-33.

4. Xu, C., Daniel, J. (2018). Wireless Temperature Sensor for High Temperature Environments (up to $1000{ }^{\circ} \mathrm{C}$ ) using RF Techniques with 0.5 meter Sensing Distance, 41th Annual Conference on Composites, Materials, and Structures, Cocoa Beach, FL.

5. Xu, C. and Daniel, J. (2017). Wireless Temperature Measurement Based on Radio Frequency (RF) Technology, 40th Annual Conference on Composites, Materials, and Structures, Cocoa beach, FL.

6. Mondal, S., and Kumar, D., "A wireless passive $\mathrm{pH}$ sensor with clutter rejection scheme," IEEE Sensors Journal, 19 (9), 3399-3407, 2019.

7. Coble, J. B., Ramuhalli, P., Bond, L. J., Hines W., and Upadhyaya B., "Prognostics and health management in nuclear power plants: a review of technologies and applications," Pacific Northwest National Lab. (PNNL), Richland, WA (United States), 2012.

8. Chahal, P., Kumar, D., Mondal, S., \& Karuppuswami, S. Systems and methods for a multiband sensing platform (2020). U.S. Patent Application No. 16/695,758.

9. Chahal, P., Mondal, S., Harmonic RFID Tag-Reader System for Long Range Sensing Identification And Security (2019). U.S. Patent Application No. 16/420,711.

10. Kumar, Deepak., Mondal, Saikat., Chahal, Premjeet., Deng, Yiming. "Wireless Battery-free Harmonic Communication" (Submitted).

11. Kumar, Deepak., Mondal, Saikat., Kaur, Amanpreet., Deng, Yiming., Chahal, Premjeet. "An RF backscatter system for multi-sensing applications." Sensors and Actuators: Physical A., 303, 111685.

12. Kumar, Deepak, Modal, Saikat, Chahal, Premjeet, Deng, Yiming, "A passive wireless harmonic communication system for pressure sensing." IEEE Sensors Letters. (Submitted).

13. R. Artmann, "Electronic identification systesm: state of the art and their further development," Comput. Electron. Arg., 24, 5-26 (1999).

14. R. Want, "Enabling ubiquitous sensing with RFID," Invisible Computing, 84-86 (2004).

15. R. A. Potyrailo and W. G. Morris, "Multianalyte chemical identification and quantitation using a single radio frequency identification sensor," Anal. Chem., 79, 45-51 (2007).

16. Umberto Cella, "Electromagnetic Wave Wireless Communication in Shallow Water Coastal Environment: Theoretical Analysis and Experimental Results," WUWNet'09, Nov 3, 2009.

17. Ahmed I. Al-Shamma'a, "Propagation of Electromagnetic Waves at MHz Frequencies Through Seawater", IEEE Transaction on Antenna and Propagation, Vol. 52, No. 11, November 2004.

18. J. H. Goh, “Underwater Wireless Communication System,” 2009, J. Phys.:Conf. Ser. 178012029.

19. Rymantas Kazys, "High Temperature Ultrasonic transducers for Imaging and Measurement in a 
Liquid $\mathrm{Pb} / \mathrm{Bi}$ Eutectic Allow," IEEE Transactions on Ultrasonics, Ferroelectrics, and Frequency Control, VOL. 52, NO. 4, April 2005.

20. Zhao, R., Shao, G., Cao, Y., An, L. and Xu, C. (2014). Temperature Sensor Made of PolymerDerived Ceramics for High-Temperature Applications. Sensors and Actuators: A. Physical, 219, 5864.

21. Freese, D., Shao, G. and Xu, C. (2013). Polymer-Derived Ceramic Sensors for Temperature Measurement in Harsh Environment. ASME Turbo Expo. Antonio, TX.

22. (Invited) Gong, X., An, L. and Xu, C. (2012). Wireless Passive Sensor Development for Harsh Environment Applications. IEEE International Workshop on Antenna Technology. Tucson, AZ.

23. Jiang, T., Hill, A., Fei, W., Wei, Y. Tellam, M., Xu, C. and An, L. (2010). Making Bulk Ceramics from Polymeric Precursors. Journal of the American Ceramic Society, 93(10), 3017-3019.

24. Zhao, R., Shao, G., Li, N., Xu, C. and An, L. (2016). Development of A Wireless Temperature Sensor Using Polymer-Derived Ceramics. Journal of Sensors, Article ID 8624817.

25. Xu, C. and Wang, K. (2017). Effect of Processing Conditions on Electric and Dielectric Properties of Polymer-Derived SiC Ceramics, Materials Science \& Technology 2017 (MS\&T 2017). Pittsburgh, PA.

26. Jia, Y., Chowdhury, A. and Xu, C., (2020). Electromagentic Property of Polymer Derived SiC-C Solid Solution Formed at Ultra-High Temperature, Carbon, Vol. 162, pp. 74-85.

27. Chowdhury, A., Wang, K., Jia, Y. and Xu, C., (2019). Semiconductor-Conduction Transition of Pristine Polymer-Derived Ceramics SiC Pyrolyzed at Temperature Range from $1200 \mathrm{oC}$ to $1800 \mathrm{oC}$, Journal of the American Ceramic Society, Vol. 103, No. 4, pp. 2630-2642.

28. Zhang, L., Wang, Y., Wei, Y., Xu, W., Fang, D., Zhai, L., Lin, K-C., An, L, 2008, “A Silicon Carbonitride Ceramic with Anomalously High Piezoresistivity”, J. Am. Ceram. Soc., 91 (4), pp. 13461349.

29. An, L., Wang, Y., Bharadwaj, L., Zhang, L., Fan, Y., Jiang, D., Chow, L.C., 2004, Silicoaluminum Carbonitride with Anomalously high resistance to oxidation and hot corrosion, Advanced Engineering Materials, 6(5), pp. 337-340.

30. Liu, J., Li, J., Shao, G., Ji, Y., Xu, C. and An, L. (2011). Investigation of Micro-End-Milling of Polymer-Derived Ceramics for High Temperature Micro-Sensor Fabrication. NSF CMMI Engineering Research and Innovation Conference. Atlanta, GA.

31. Liu, J., Li, J., Grummel, B., Xu, C. and Shen, J., 2010, “A Contactless Polymer Derived Ceramic Temperature Sensing System for Turbine Applications”, Florida Center for Advanced AeroPropulsion (FCAAP) - Annual Technical Symposium.

32. Liu, J., Xu, C. and An, L. (2010). Micro-machinable Polymer-Derived Ceramics Sensors for HighTemperature Applications. ASME Conference on Smart Materials, Adaptive Structures and Intelligent Systems. San Diego, CA.

33. Shao, G., Freese, D., Xu, C. and An, L. (2013). Polymer Derived Ceramic Sensors for Ultra-High Temperature Application. 37th International Conference and Exposition on Advanced Ceramics and Composites, Combustion Institute, Daytona, FL.

34. X. Gong, L. An, and Xu, C. (2011), "Recent advances on wireless passive high-temperature sensors for harsh environments," in 35th International Conference \& Exposition on Advanced Ceramics and Composites, Daytona Beach, FL. (Invited)

35. Ding-Xin Yang, Zheng Hu, Hong Zhao, Hai-Feng Hu, Yun-Zhe Sun and Bao-Jian Hou, "Through- 
Metal-Wall Power Delivery and Data Transmission for Enclosed Sensors: A Review," Sensors 2015, 15, 31581-31605. doi:10.3390/s151229870

36. Imoru, O.; Jassal, A.; Polinder, H.; Nieuwkoop, E.; Tsado, J.; Jimoh, A.A. "An Inductive Power Transfer through metal object," In Proceedings of the 2013 1st International Future Energy Electronics Conference, (IFEEC), Tainan, Taiwan, 3-6 November 2013; pp. 246-251.

37. Zangl, H.; Fuchs, A.; Bretterklieber, T.; Moser, M.J.; Holler, G., "Wireless Communication and Power Supply Strategy for Sensor Applications Within Closed Metal Walls," IEEE Trans. Instrum. Meas. 2010, 59, 1686-1692.

38. Yamakawa, M.; Mizuno, Y.; Ishida, J.; Komurasaki, K.; Koizumi, H., "Wireless Power Transmission into a Space Enclosed by Metal Walls Using Magnetic Resonance Coupling." Wirel. Eng. Technol., 2014, 5, 19-24.

39. Graham, D.J. "Investigation of Methods for Data Communication and Power Delivery through Metals," Ph.D. Thesis, University of Newcastle upon Tyne, Newcastle upon Tyne, UK, 2012.

40. WFS, Seatooth RF (Radio Frequency) communication, https://www.wfs-tech.com/wireless-smartdevices-rental/

41. Jorge V. Carvajal, Shawn C. Stafford, Jeffrey L. Arndt, Paul M. Sirianni, Melissa M. Heagy, Emre Tatli, David M. Carpenter, Yakov Ostrovsky, "Integral Fuel Rod Real-Time Wireless Sensor," ANS, 11th Nuclear Plant Instrumentation, Control and Human-Machine Interface Technologies, Renaissance Orlando at SeaWorld, Orlando, FL, February 9-14, 2019

42. Jorge V. Carvajal, Jeffrey L. Arndt, Shawn C. Stafford, Detection Apparatus Usable In A Nuclear Reactor And Associated Method, US 2019 / 0180884 A1, Jun 13, 2019

43. Jorge V. Carvajal, Shawn C. Stafford, Michael D. Heibel, Paul M. Sirianni, Melissa M. Heagy, Robert W. Flammang, Nicola G. Arlia, James A. Turso, Kenan Unlu, "Integral Fuel Rod Real-Time Wireless Sensor", ANS, 11th Nuclear Plant Instrumentation, Control and Human-Machine Interface Technologies, Renaissance Orlando at SeaWorld, Orlando, FL, February 9-14, 2019

44. John G. Seidel, Robert W. Flammang, Jorge V. Carvajal, Michael A. James, Nicola G. Arlia, "Wireless In-Core Neutron Monitor," Patent No. US 8,767,903 B2.

45. Edward Mercer, "What Is a Tank Circuit?" https://www.techwalla.com/articles/what-is-a-tank-circuit.

46. Susquehanna Nuclear Energy Guide, http://www.pplweb.com/NR/rdonlyres/F63D7386-A57E-46C690A5-857D513B0254/0/seic plantguide.pdf.

47. Boiling Water Reactor (BWR) Systems, Reactor Concepts Manual, USNRC Technical Training Center, https://www.nrc.gov/docs/ML1209/ML120970422.pdf.

48. Containment Building, https://en.wikipedia.org/wiki/Containment building.

49. Westinghouse Technology Systems Manual, Section 5.3 Containment, USNRC HRTD, https://www.nrc.gov/docs/ML1122/ML11223A221.pdf.

50. Nuclear Plant Security Systems, PDH Course E182, http://www.pdhcenter.com/courses/e182/e182content.pdf.

51. Boiling water reactor figure courtesy of USNRC, https://www.nrc.gov/reactors/bwrs.html\#: :text=Typical\%20Boiling\%2DWater\%20Reactor\&text=In $\% 20 \mathrm{a} \% 20$ typical $\% 20$ design $\% 20$ concept,through $\% 20$ the $\% 20$ core $\% 2 \mathrm{C} \% 20$ absorbing $\% 20$ heat.

52. Plant Vogtle's containment building, https://www.southerncompany.com/our-companies/southernnuclear/southern-nuclear-news-stories/nuclear- 
containment.html\#: :text=Steel $\% 2$ Dreinforced $\% 20$ concrete $\% 20$ containment $\% 20$ buildings,diameter \%20and $\% 20226 \% 20$ feet $\% 20$ high

53. Xiaoning Jiang, Mohamed Bourham, Mo-Yuen Chow, and Leigh Winfrey, NC State, "High Temperature Embedded/Integrated Sensors (HiTEIS) for Remote Monitoring of Reactor and Fuel Cycle Systems," https://www.energy.gov/sites/prod/files/2018/07/f53/2018\%20Advanced\%20Sensors\%20and\%20Inst rumentation $\% 20$ Award $\% 20$ Summaries.pdf.

54. Richard B. Vilim, Alexander Heifetz, Argonne National Laboratory, "Transmission of Information by Acoustic Communication along Metal Pathways in Nuclear Facilities," https://www.energy.gov/sites/prod/files/2017/06/f34/2017\%20Advanced\%20Sensors\%20and\%20Inst rumentation $\% 20$ Award $\% 20$ Summaries.pdf.

55. Lei Zuo, Dong Ha, Virginia Tech, Haifeng Zhang, University of North Texas, Roger Kisner, Oak Ridge National Laboratory, "Self-Powered Wireless Through-Wall Data Communication For Nuclear Environments," https://www.energy.gov/sites/prod/files/2017/06/f34/2017\%20Advanced\%20Sensors\%20and\%20Inst rumentation $\% 20$ Award $\% 20$ Summaries.pdf.

56. In-Pile Instrumentation Initiative: A Multidisciplinary Scientific Approach For Characterization Of Fuels And Materials, INL/EXT-17-43366, 01-SEP-17.

57. James A. Smith, Dale K. Kotter, Rick O. Parry, "Thermo-Acoustic Sensor for In-Situ Nuclear Reactor Microstructure and Process Monitoring," FCRD program, INL/LTD-12-26875, August 28, 2012.

58. S. Garrett, et al., "Fission-powered in-core thermoacoustic sensor," Appl. Phys. Lett. 108, 144102 (2016).

59. Garrett, Steven L., Smith, James A., Smith, Robert W., Heidrich, Brenden J., Heibel, Michael D., "Using the Sound of Nuclear Energy," American Nuclear Society's Nuclear Technology, Vol. 195, No. 3, PP. 353-362, September, 2016.

60. S. L. Garrett, J. A. Smith, and D. K. Kotter, "Thermoacoustic Enhancements for Nuclear Fuel Rods," U.S. Pat. No. 10,283,225 (May 7, 2019).

61. S. L. Garrett, J. A. Smith, and D. K. Kotter, "Thermoacoustic Enhancements for Nuclear Fuel Rods and other High Temperature Applications,” U.S. Pat. No. 9,646,723 (May 9, 2017).

62. M. D. Heibel, R. W. Flammang, and D. M. Sumego, "Thermo-Acoustic Nuclear Power Distribution Measurement Assembly,” U.S. Pat. No. 9,287,014 (Mar. 15, 2016).

63. S. Garrett and D. Brown, "Gamma Thermoacoustic Sensor," U.S. Provisional Pat. Appl. No. 62,818,824 (Mar. 15, 2019).

64. S.W. Glass, A.M. Jones, L.S. Fifield, and T.S. Hartman, "Bulk and Distributed Electrical Cable NonDestructive Examination Methods for Nuclear Power Plant Cable Aging Management Programs," Light Water Reactor Sustainability Program, PNNL-25634, September 2016

65. EPRI, "Plant Engineering: Evaluation and Insights from Nuclear Power Plant Tan Delta Testing and Data Analysis - Update," TR-3002005321, Electric Power Research Institute, Palo Alto, California, 2015.

66. Mantey A. "Evaluation and Insights from Nuclear Power Plant Tan Delta Testing and Data AnalysisUpdate," Presented at IEEE Proceedings of the Power and Energy Society Insulated Conductors Committee, Tuscon, Arizona, November 1-4, 2015. 
67. CommScope Coaxial Drop Cable, 7451203 | F677TSVV XP, https://www.commscope.com/producttype/cables/coaxial-cables/drop-cables/item7451203/.

68.

Lowe, C. L., C. J. Kiger, D. N. Jackson, and D. M. Young, 2017, "Implementation of Wireless Technologies in Nuclear Power Plants' Electromagnetic Environment Using Cognitive Radio System," NPIC\&HMIT, San Francisco, California, June 11-15, 2017.

69.

Barker, B., 2017, “A Wireless Eye in Nuclear Plants," EPRI Journal, http://eprijournal.com/a-wireless-eye-in-nuclear-plants/, July 25, 2017.

70.

Manjunatha, K. A., and V. Agarwal, "Review of Wireless Communication Technologies and Techno-Economic Analysis," Idaho National Laboratory, INL/EXT-19-53966, May 2019.

71. SOLiD, 2017, “Alliance MultiOperator DAS 5W Mid-Power Remote Optic Unit," https://solid.com/wordpress/wpcontent/uploads/SOLiD-Data-Sheet-ALLIANCE-5W-Remote-Unit-MROU-v2.7.pdf, September 2017.

72.

R. F. Globalnet, "Distributed

Antenna System (DAS) for IoT, Cellular, and Other Wireless Applications," Website:

https://www.rfglobalnet.com/doc/distributed-antenna-system-das-for-iot-cellular-and-other-wirelessapplications-0001.

73. Khorov, E., A. Kiryanov, A. Lyakhov, and G. Bianchi, 2018, "A tutorial on IEEE 802.11 ax high efficiency WLANs,” IEEE Communications Surveys and Tutorials, Vol. 21, No. 1, pp. 197-216.

74. URL: $\underline{\text { https://www.cisco.com/c/dam/en/us/products/collateral/wireless/white-paper-c11-740788.pdf }}$

75. De Carvalho Silva, J., et al., "LoRaWAN-A low power WAN protocol for Internet of Things: A review and opportunities," 2017 2nd International Multidisciplinary Conference on Computer and Energy Science (SpliTech), IEEE, 2017.

76. Klair, D. K., K. W. Chin, R. A, Raad, "Survey and Tutorial of RFID Anti-Collision Protocols," IEEE Commun. Surv. Tutor., 2010, Vol. 12, pp. 400-421. 